ACCEPted For publication in the Astrophysical Journal

Preprint typeset using $\mathrm{LAT}_{\mathrm{E}} \mathrm{X}$ style emulateapj v. 08/22/09

\title{
THE LUMINOSITY DEPENDENCE OF THE GALAXY MERGER RATE
}

\author{
D. R. PATton ${ }^{1}$ AND J. E. Atfield ${ }^{1}$ \\ Accepted for publication in the Astrophysical Journal
}

\begin{abstract}
We measure the number of companions per galaxy $\left(N_{c}\right)$ as a function of $r$-band absolute magnitude for both the Sloan Digital Sky Survey and the Croton et al. (2006) semi-analytic catalog applied to the Millennium Run simulation. For close pairs with projected separations of $5-20 h^{-1} \mathrm{kpc}$, velocity differences less than $500 \mathrm{~km} \mathrm{~s}^{-1}$, and luminosity ratios between 1:2 and 2:1, we find good agreement between the observations and simulations, with $N_{c}$ consistently close to 0.02 over the range $-22<$ $M_{r}<-18$. For larger pair separations, $N_{c}\left(M_{r}\right)$ instead becomes increasingly steep towards the faint end, implying that luminosity-dependent clustering plays an important role on small scales. Using the simulations to assess and correct for projection effects, we infer that the real-space $N_{c}\left(M_{r}\right)$ for close pairs peaks at about $M^{*}$, and declines by at least a factor of two as $M_{r}$ becomes fainter. Conversely, by measuring the number density of close companions, we estimate that at least $90 \%$ of all major mergers occur between galaxies which are fainter than $L^{*}$. Finally, measurements of the luminosity density of close companions indicate that $L^{*}$ galaxies likely dominate in terms of the overall importance of major mergers in the evolution of galaxy populations at low redshift.
\end{abstract}

Subject headings: galaxies: evolution, galaxies: interactions, surveys, galaxies: statistics

\section{INTRODUCTION}

Galaxy mergers can produce dramatic changes in the morphological, nuclear and star forming properties of galaxies over relatively short timespans. As a result, mergers have been invoked to explain a number of aspects of galaxy evolution. In recent years, large redshift surveys have paved the way for systematic observational studies of candidate mergers, while theoretical modelling of structure formation has produced key advances in our understanding of the role of merging in a cosmological context. In general, these efforts have focussed on two key aspects of merging: (1) the effects of merging on the constituent galaxies, and (2) the frequency with which merging occurs, as described by the merger rate and related quantities.

An increasingly popular method of identifying candidate merging systems is the use of close galaxy pairs, which are the precursors to mergers. With careful choices of close pair criteria, and correction for projection effects (ie., contamination by non-merging pairs), the frequency of close pairs should correlate with the merger rate. Recent studies using cosmological simulations support this idea, demonstrating that most close pairs merge on relatively short timescales (Kitzbichler \& White 2008). In addition, the properties of paired galaxies can provide insight into the nature of merging galaxies both before and during the encounter. Galaxies in close pairs have higher asymmetries than galaxies in wider separation pairs or the field (Hernández-Toledo et al. 2005; Patton et al. 2005; De Propris et al. 2007), confirming that interactions and mergers are prevalent in these systems. Star formation is enhanced in close pairs at low redshift (Lambas et al. 2003; Alonso et al. 2004; Nikolic et al.

\footnotetext{
${ }^{1}$ Department of Physics \& Astronomy, Trent University, 1600 West Bank Drive, Peterborough, ON, K9J 7B8, Canada; dpatton@trentu.ca, julianatfield@trentu.ca
}

2004; Patton et al. 2005; Sol Alonso et al. 2006; Geller et al. 2006; Barton et al. 2007; Owers et al.|2007; Smith et al. 2007; Woods \& Geller 2007; Ellison et al. 2008; Li et al. 2008), implying that star formation has been triggered by mergers or interactions. Differences in metallicities between paired and field galaxies are consistent with a scenario in which interactions funnel gas to the central regions of galaxies involved in these close encounters (Kewlev et al. 2006; Ellison et al. 2008). Most ultraluminous infrared galaxies (ULIRGs) originate from major mergers of gas rich galaxies (Dasyra et al. $2006 \mathrm{a}, \mathrm{b})$, while approximately half of the luminous infrared galaxies (LIRGs) at low redshift appear to be undergoing interactions or mergers (Wang et al. 2006).

Using close galaxy pairs and/or galaxy asymmetries as indicators of imminent or recent mergers, the merger rate and its evolution has now been measured using a number of large redshift surveys (Carlberg et al. 2000; Le Fèvre et al. 2000; Patton et al. 2002; Conselice et al. 2003; Bundy et al. 2004; Lin et al. 2004; Bell et al. 2006a; Kartaltepe et al. 2007; Kampczyk et al. 2007; Conselice et al. 2008; Hsieh et al. 2008; Lin et al. 2008; Lotz et al. 2008; Rawat et al. 2008; Ryan et al. 2008). Evolution estimates range from roughly $(1+z)^{0.5}$ to $(1+z)^{3}$, implying widely differing scenarios at $z \sim 1$ and above. At least some of these discrepancies result from the use of different pair criteria. For example, simulations and semi-analytical models of galaxy formation indicate that the merger rate and its evolution depends on factors such as galaxy mass, pair mass ratio and environment (Khochfar \& Burkert 2001; Berrier et al. 2006; Maller et al. 2006; Cox et al. 2008; Guo \& White 2008; Kitzbichler \& White 2008).

In order to better understand the role of merging, and to reconcile merger rate measurements from disparate samples, we need to assess how the frequency and nature of merging depends on factors such as environment, mass ratio (ie., major versus minor mergers), 
properties of the progenitor galaxies (e.g., dry mergers versus gas-rich mergers), and the mass (or luminosity) of the merging galaxies or merger remnants (e.g., formation of massive galaxies versus $L^{*}$ galaxies). Significant observational progress has been made in all of these areas in recent years. Galaxy groups appear to be an ideal environment for mergers (Goto 2005; Brough et al. 2006; Miles et al. 2006; Robotham et al. 2006; Zandivarez et al. 2006; Coziol \& Plauchu-Fravn 2007; Nolan et al. 2007), though the infall regions of clusters (van Dokkum et al. 1999; Tran et al. 2005; Moss 2006) and the low density field (Barton et al. 2007) are important too. Induced star formation appears to be strongest in major mergers, or in the lower luminosity (or mass) members of minor mergers (Woods et al. 2006; Woods \& Geller 2007; Ellison et al. 2008). Dry mergers have been invoked to explain the assembly of massive elliptical galaxies since $z \sim 1$ van Dokkum 2005; Bell et al. 2006b; Naab et al. 2006), though this process likely cannot explain all recently formed early type galaxies (Cox et al. 2006; Brown et al. 2007; Bundy et al. 2007; Scarlata et al. 2007).

In this paper, we investigate the luminosity dependence of the merger rate at low redshift, using close galaxy pairs in the Sloan Digital Sky Survey (York et al. 2000), and in the Croton et al. (2006) semi-analytic galaxy catalogs derived from the Millennium Run simulation (Springel et al. 2005). Both of these samples are large enough that, in addition to being able to measure close pair statistics as a function of absolute magnitude, we also have the luxury of being very selective in how we choose our close pairs. In particular, we require all of our pairs to have spectroscopic redshifts for both members, projected separations less than $20 h^{-1} \mathrm{kpc}$, and relative velocities less than $500 \mathrm{~km} \mathrm{~s}^{-1}$. In addition, we require our companion sample to be volume limited for all luminosity ratios between $1: 2$ and $2: 1$, thereby providing a cleaner match to the major merger candidates we seek to identify. Moreover, our measurements are carried out in the $r$-band, yielding absolute magnitudes that are a better proxy for stellar mass than those at shorter wavelengths. This is beneficial for close pair studies, since merger-induced star formation is likely to affect the luminosities of galaxies in pairs more than normal (isolated) galaxies.

We begin by describing the creation of our SDSS spectroscopic and photometric samples in $\S 2$. Section 3 outlines the calculation of the number of close companions per galaxy $\left(N_{c}\right)$ for SDSS, including corrections for spectroscopic incompleteness. We make a direct comparison with the Millennium Run simulation in $\S$ 4, and derive real space pair statistics for both SDSS and Millennium in $\S$ 5. With additional assumptions, we then relate these close pair statistics to the merger rate in $\S$. We summarize our conclusions in $\S 7$. Throughout this study, we adopt cosmological parameters of $\Omega_{m}=0.3, \Omega_{\Lambda}=0.7$, and $H_{0}=100 h \mathrm{~km} \mathrm{~s}^{-1} \mathrm{Mpc}^{-1}$. For brevity, we express all absolute magnitudes as $M_{r}$ instead of $M_{r}-5 \log (h)$.

\section{DATA}

The fifth data release (DR5) of the Sloan Digital Sky Survey (hereafter SDSS) consists of ugriz imaging spanning over $8000 \mathrm{deg}^{2}$, along with spectra of about one million galaxies, quasars, and stars within a $5713 \mathrm{deg}^{2}$ subset of the imaging area Adelman-McCarthy et al. 2007). In this study, we use the main galaxy sample, as described by Strauss et al. (2002). Unlike the luminous red galaxy sample of Eisenstein et al. (2001), this sample is designed to be independent of galaxy luminosity and Hubble type, and therefore probes a representative sample of galaxies, including both gas-rich and gas-poor ("dry") merger candidates.

Our goal is to carry out a census of galaxies with close physical companions. We restrict our observational sample to close galaxy pairs for which redshifts are available for both galaxies. This reduces contamination due to non-merging systems, and allows one to measure restframe properties of the pairs and their member galaxies. However, the SDSS is not a complete spectroscopic sample, and in fact the minimum fiber separation of $55^{\prime \prime}$ biases the sample against the close pairs we are interested in. Fortunately, it is possible to measure and correct for spectroscopic incompleteness by using the photometry of all galaxies, regardless of whether or not spectra were obtained. To this end, we now describe the creation of both spectroscopic and photometric catalogs of galaxies drawn from the main galaxy sample.

\subsection{Spectroscopic Catalog}

The SDSS main galaxy sample consists of galaxies with $r$-band Petrosian magnitudes of $m_{r} \leq 17.77$ (Strauss et al. 2002), after correction for Galactic extinction. However, regions covered by the first data release of the survey had limiting apparent magnitudes ranging from 17.5 to 17.77 (Abazajian et al. 2003). In addition, the sample becomes incomplete at the bright end, where automated deblending of large galaxies becomes unreliable (Strauss et al. 2002), and introduces concerns about single galaxies mistakenly being classified as close pairs. To ensure a complete and reliable sample, we therefore begin by restricting our catalogs to the range $14.5 \leq m_{r} \leq 17.5$. We also ensure that every galaxy has a measured spectroscopic redshift; moreover, we require the SDSS "zConf" parameter to be at least 0.7, thereby ensuring that the confidence in each redshift measurement is at least $70 \%$ (in most cases, it is much higher). Using these criteria, we create a preliminary spectroscopic sample by querying the SDSS online database ${ }^{2}$.

For every galaxy in the spectroscopic sample, we measure the $r$-band absolute magnitude at redshift $z$, as given by

$$
M_{r}=m_{r}-5 \log d_{L}(z)-25-k_{r}-E(z),
$$

where $m_{r}$ is the extinction-corrected Petrosian $r$-band magnitude, $d_{L}$ is the luminosity distance, and $k_{r}$ and $E(z)$ are the $k$-corrections and passive stellar evolution corrections, respectively. We measure $k$-corrections using the SDSS ugriz photometry, employing the kcorrect software (version v1_1_4) of Blanton \& Roweis (2007). Following Patton et al. (2002), we parameterize the evolution correction as $E(z)=-Q z$, where $Q$ is determined from measurements of the galaxy luminosity function (LF) and $z$ is the redshift (Lin et al. 1999). We adopt $Q=1.8$ as an average of the SDSS ${ }^{0.1} r$ and ${ }^{0.1} g Q$ measurements of Blanton et al. (2003a), since rest-frame $r$

\footnotetext{
${ }^{2}$ http://casjobs.sdss.org/CasJobs/
} 
lies between these two passbands (this agrees well with the evolutionary correction of Tegmark et al. (2004)). Given the relatively low redshift of our sample, however, this evolution correction is small, and removing it entirely (ie., $Q=0$ ) does not significantly change any of the conclusions in this paper.

\subsection{Photometric Catalog}

In order to measure and correct for incompleteness in our spectroscopic catalog, we also create a photometric catalog, in which galaxies satisfy the same flux limits as the spectroscopic catalog, but are not required to have a measured redshift. The sky area covered by DR5 imaging is about $40 \%$ larger than the area with spectroscopic coverage (Adelman-McCarthy et al. 2007); to match the footprints of our spectroscopic and photometric catalogs, we therefore also require every galaxy in both samples to have at least one galaxy with a spectrum within 12 arcminutes of its position (excluding itself).

\subsection{Spectroscopic Incompleteness}

By comparing our resulting photometric and spectroscopic catalogs, we find that the average spectroscopic completeness is $88 \%$. While spectroscopic target selection was designed to provide uniform coverage (Blanton et al. 2003b), the completeness does vary considerably from one part of the sky to another, with the completeness in some regions falling well below the mean, while in other regions (particularly those covered by more than one SDSS plate), the completeness is considerably higher. Patton et al. (2002) demonstrated that the observed number of companions per galaxy scales with the spectroscopic completeness. While this bias can be corrected for, one can also minimize its impact by excluding regions with low spectroscopic completeness. Such an exclusion will also remove galaxies which lie close enough to the survey boundaries that close companions will be missed. With this in mind, and with a desire to measure and correct for the remaining spectroscopic incompleteness, we compute a measure of local spectroscopic completeness for every galaxy in our spectroscopic and photometric catalogs. We consider an area around each galaxy within an outer radius of one degree, and an inner radius of $55^{\prime \prime}$ (the latter corresponds to the minimum fiber separation). After counting the number of enclosed galaxies in the spectroscopic and photometric samples, we take the ratio of these two numbers, denoting this quantity $f_{s}$. We then require every galaxy in our cata$\log$ s to have $f_{s}>0.75$. This restriction excludes $4.7 \%$ of the galaxies in the spectroscopic catalog. The main conclusions of this paper are unchanged if we instead use $f_{s}>0.7$ or $f_{s}>0.8$ (excluding $2.5 \%$ and $9.5 \%$ of the spectroscopic catalog respectively).

\section{SDSS CLOSE PAIR STATISTICS}

\subsection{Methodology}

We now proceed to identify close galaxy pairs from our spectroscopic sample. We measure three key quantities for each galaxy pair: projected physical separation $r_{p}$, rest-frame relative velocity $\Delta v$, and absolute magnitude difference $\Delta M_{r}$. Following Patton et al. (2000, 2002), we define a "close pair" to have $5<r_{p}<20 h^{-1}$ $\mathrm{kpc}$ and $\Delta v<500 \mathrm{~km} \mathrm{~s}^{-1}$. On order half of the pairs satisfying these criteria are known to exhibit morphological signs of interactions, based on visual classification (Patton et al. 2000) and quantitative measures of asymmetry (Patton et al. 2005; De Propris et al. 2007). In addition, in order to focus on major merger candidates, we require that $\left|\Delta M_{r}\right| \leq 0.753$, ensuring a pair luminosity ratio between $1: 2$ and $2: 1$. This criterion is preferable to the more common approach of selecting both host and companion galaxies from the same fixed range in absolute magnitude (e.g., Patton et al. (2000, 2002); Lin et al. (2004); De Propris et al. (2005)), since that approach includes a wider range in luminosity ratios, and becomes increasingly incomplete at luminosity ratios significantly different from 1:1.

\subsection{Choosing Potential Host and Companion Galaxies}

Figure 1 contains a plot of absolute magnitude versus redshift for 10,000 galaxies selected at random from our spectroscopic sample of about 337,000 galaxies. We begin our sample selection by measuring the bright and faint limits in absolute magnitude as a function of redshift within which galaxies of all spectral types will have $14.5 \leq m_{r} \leq 17.5$. These are shown by the upper and lower dashed (black) curves in Figure 1 and are computed using estimates of the minimum and maximum $\mathrm{k}$-corrections respectively. We denote all galaxies lying within these limits as potential companion galaxies.

Within this sample, we then identify the subset of galaxies for which all companions (with $\left|\Delta M_{r}\right| \leq 0.753$ ) are detectable. These galaxies, which we refer to as potential host galaxies, are contained within the two solid (red) curves in Figure 1. Aside from spectroscopic incompleteness (which we correct for statistically), this provides a sample of host galaxies for which we can carry out a volume limited search for companions. It follows that our sample will contain close pairs in which either one or both galaxies fall into the sample of potential hosts.

We wish to compute pair statistics as a function of $M_{r}$, over as large a range as feasible. However, in order to ensure completeness, we must impose some restrictions on the range of host galaxy luminosities. At the bright end, Figure11indicates that our sample contains relatively few galaxies close to $M_{r} \sim-23$. Given the need to detect companions which are 0.753 magnitudes brighter than any potential host galaxy, we therefore impose a minimum $M_{r}=-22$. At the faint end, the key issue is to decide on a reasonable minimum redshift to use, as this will dictate a maximum allowable absolute magnitude. Galaxies at the lowest redshifts have the least certain absolute magnitudes, due to the increased influence of peculiar velocities on the observed redshift. In addition, such nearby galaxies are typically the most challenging to obtain accurate photometry for, since the SDSS deblending algorithm tends to break down more often for galaxies with large apparent sizes. With these considerations in mind, we impose a conservative maximum $M_{r}$ of -18 on our sample of potential host galaxies, corresponding to a minimum redshift of 0.015 . Companions are permitted to lie at slightly lower redshifts due to the allowed velocity difference of $500 \mathrm{~km} \mathrm{~s}^{-1}$.

With these criteria, we find a total of 477 host galaxies which have at least one close companion. In order to ensure that the SDSS pipeline has been successful in detecting real galaxy pairs, we visually inspect the SDSS 
images of every detected host galaxy. The contamination was found to be very small, with only $0.8 \%$ of the host galaxies being spurious. The affected systems consist of one edge-on disk, and one ring galaxy: in both cases, the SDSS pipeline mistakenly detected two galaxies. We therefore remove these galaxies from our sample, leaving 473 host galaxies in the range $-22<M_{r}<-18$. The basic properties of these galaxies are listed in Table 1 (this table contains a subset of the table, which is to be published in its entirety in the electronic version of the journal).

A significant fraction of these galaxies exhibit morphological signs of interactions, though we defer a more rigorous structural analysis to a future paper. We note that this sample is more than twice as large as the Millennium Galaxy Catalogue sample of De Propris et al. (2005), despite our more rigid requirement that the sample be volume-limited for close companions which are major merger candidates $\left(\left|\Delta M_{r}<0.753\right|\right)$.

\subsection{Small Scale Spectroscopic Incompleteness}

In Section 2.3, we described our algorithm for measuring the local spectroscopic completeness for every galaxy. This provides a measure of completeness on scales of on order half a degree. However, constraints on fiber placement require angular separations of at least $55^{\prime \prime}$ between any two targets assigned to the same plate (Strauss et al. 2002). As a result, spectroscopic completeness drops sharply at smaller angular pair separations. At a redshift of $0.1,55^{\prime \prime}$ corresponds to a projected separation of 71 $h^{-1} \mathrm{kpc}$, meaning that most of the close pairs of interest in this study $\left(r_{p}<20 h^{-1} \mathrm{kpc}\right)$ are found at these small angular separations. Fortunately, most plates contain regions of overlap with adjacent plates, and some regions are observed using two or more plates. As a result, the spectroscopic sample contains enough close angular pairs that we are able to model the incompleteness and correct for it, following the method of Patton et al. (2002).

First, we measure the ratio of spectroscopic to photometric pairs $\left(N_{z z} / N_{p p}\right)$ as a function of angular separation $\theta$, as shown in the upper panel of Figure 2. For a fair sample, in which spectroscopic completeness is independent of pair separation, one would expect to find $N_{z z} / N_{p p} \sim f_{s}^{2}$, where $f_{s}$ is the overall spectroscopic completeness of the survey. Instead, we see a sharp drop in $N_{z z} / N_{p p}$ below $55^{\prime \prime}$, as expected. We model this incompleteness by fitting a function $g(\theta)$ to these data. We then multiply each spectroscopic pair by a weight $w_{\theta}=f_{s}^{2} / g(\theta)$. The resulting corrected values of $N_{z z} / N_{p p}$ are plotted in the lower panel of Figure 2. It is clear that this weighting scheme is successful in removing the angular dependence of the small scale spectroscopic incompleteness, with the corrected $N_{z z} / N_{p p} \approx f_{s}^{2}$ at all relevant angular separations, to within the reported errors. Therefore, by applying $w_{\theta}$ weights to each detected pair, we can remove this very significant bias from our measurements.

\section{4. $N_{c}\left(M_{r}\right)$ for SDSS Pairs}

We now have a catalog of host galaxies for which we can measure close pair statistics, using statistical weights to correct for spectroscopic incompleteness. Weights are combined using the approach outlined by Patton et al.
(2002). First, the number of companions for galaxy $i$, summed over all companions $j$, is given by

$$
N_{c_{i}}=\sum_{j} f_{s_{j}}^{-1} w_{\theta_{i j}} .
$$

The statistical weights in this equation are used to correct the observed number of companions to the number that would have been observed in a complete redshift survey. The mean number of companions per galaxy, weighted by spectroscopic completeness, is then given by

$$
N_{c}=\frac{\sum_{i} f_{s_{i}} N_{c_{i}}}{\sum_{i} f_{s_{i}}} .
$$

This weighting scheme places greater importance on galaxies in regions of higher spectroscopic completeness, thereby minimizing statistical uncertainties.

The resulting measurements of $N_{c}\left(M_{r}\right)$, calculated using equations 2 and 3 , are indicated by the solid (blue) curve in Figure 3. In addition, to facilitate future comparisons with other surveys, we provide these results in the first row of Table 2. We find that $N_{c}\left(M_{r}\right)$ is approximately constant over the given range in absolute magnitude, with a mean of $N_{c}=0.021 \pm 0.001$. Given that few galaxies have more than one close companion, this implies that $\sim 2 \%$ of galaxies with $-22<M_{r}<-18$ have a close companion, independent of $M_{r}$.

Overall, our mean value of $N_{c}$ agrees quite well with related measurements in the literature. For example, using identical $r_{p}$ and $\Delta v$ criteria, but no limits on luminosity ratio, Patton et al. (2000) find $N_{c}\left(-21 \leq M_{B} \leq-18\right)=$ $0.0226 \pm 0.0052$ at $z=0.015$, while De Propris et al. (2005) find $N_{c}=0.0357 \pm 0.0027$ at $z=0.116$. Given our additional requirement that companions be within a factor of two in luminosity of their host galaxy, both results are broadly consistent with the somewhat lower $N_{c}$ that we find. Our mean $N_{c}$ appears to be several times larger than the SDSS pair fraction of Kartaltepe et al. (2007). However, their close pair criteria are substantially different from ours; in particular, they require $M_{V}>-20$ for both members, they do not require spectroscopic redshifts for both members of their pairs, and their sample is derived from the Allam et al. (2004) sample of merging pairs, which have a number of additional criteria imposed (including isolation). A meaningful comparison with Kartaltepe et al. (2007) is therefore not feasible.

The flat shape of $N_{c}\left(M_{r}\right)$ that we find is a surprising result. Based on the LF alone, one would expect to find more companions (of comparable luminosity) close to intrinsically faint galaxies, since dwarf galaxies are much more numerous than giants. In other words, $N_{c}$ should be proportional to the number density of galaxies (Berrier et al. 2006). However, the number of companions per galaxy is sensitive to both number density and clustering strength (Patton et al. 2000; Berrier et al. 2006). Clustering strength is known to increase with luminosity (Norberg et al. 2001, 2002; Zehavi et al. 2002; Li et al. 2006). This provides a competing effect, which must be comparable in size to the density effect if it is to explain the flat $N_{c}\left(M_{r}\right)$ that we find. However, at separations of $100 h^{-1} \mathrm{kpc}$, the correlation function measurements of Li et al. (2006) indicate that the clustering strength of SDSS galaxies is roughly independent of absolute magnitude for $M_{r} \gtrsim-21$, and is approximately 
twice as high in the range $-22<M_{r}<-21$. In order to explain the roughly flat $N_{c}\left(M_{r}\right)$ we detect over the range $-22<M_{r}<-18$, the luminosity dependence of clustering must be considerably stronger on the smaller scales relevant for our close pairs $\left(\sim 10 h^{-1} \mathrm{kpc}\right)$. However, we must also rule out any potential luminosity-dependent biases in our sample, which could in principle contribute.

It is possible to break the degeneracy between density and clustering effects by considering wider separation pairs, since clustering strength diminishes with pair separation. We therefore compute $N_{c}\left(M_{r}\right)$ as a function of pair separation out to $100 h^{-1} \mathrm{kpc}$ (see Figure 3 and Table 22). As expected, $N_{c}\left(M_{r}\right)$ steepens towards the faint end as pair separation increases. This implies that $N_{c}\left(M_{r}\right)$ scales with number density on large scales, but flattens out on small scales as a result of the added effects of luminosity-dependent clustering. We conclude that the roughly flat $N_{c}\left(M_{r}\right)$ that we observe for close pairs is seen only on the small scales relevant for galaxy interactions and mergers, and is unlikely to be due to any overall luminosity-dependent bias within our sample.

\section{MILLENNIUM CLOSE PAIR STATISTICS}

Given the somewhat surprising trends seen in our SDSS pair statistics, and to facilitate comparison with theoretical models of galaxy formation and evolution, we now apply our techniques to a sample drawn from the semi-analytic galaxy catalogs of Croton et al. (2006), which were created using the output of the Millennium Run simulation (Springel et al. 2005). These catalogs have been shown to reproduce the overall properties of galaxies in the local universe, including the luminosity function, the two point correlation function, and the pairwise velocity dispersion (Croton et al. 2006; Li et al. 2007). The simulations provide the additional benefit of three dimensional positions and velocities, which we will use to assess and remove projection effects. While the reader is referred to Croton et al. (2006) and references therein for a detailed description of these catalogs, we note that merging is treated by following dark matter subhalos down to a mass limit of $1.7 \times 10^{10} h^{-1} M_{\odot}$, and then using the dynamical friction formula of Binney and Tremaine (1987) to estimate the remaining time until a merger takes place.

For this analysis, we use the Croton et al. (2006) ugriz catalog, which is complete for galaxies more luminous than $M_{r}=-16.6$. The stated resolution is $5 h^{-1} \mathrm{kpc}$, which conveniently coincides with our minimum pair separation criterion. We confirm, however, that the twopoint galaxy correlation function has the expected power law form to below this level, so there should not be any unexpected effects near the resolution limit.

\subsection{Mock Redshift Catalogs}

The Croton et al. (2006) catalogs contains three dimensional positions and velocities for approximately 9 million galaxies at redshift zero, within a cube which is $500 h^{-1} \mathrm{Mpc}$ on a side. In order to make a direct comparison between Millennium and SDSS, it is necessary to transform this real-space catalog into a mock redshift catalog. To this end, we begin by placing the observer at the origin of the Millennium cube, and then computing the corresponding right ascension, declination, redshift, and apparent magnitude of each galaxy in the sample.
In order to match our observed SDSS sample, we then apply the same flux limits $\left(14.5<m_{r}<17.5\right)$. In addition, we impose a minimum redshift of 0.022 , to ensure that we sample only galaxies which are more luminous than $M_{r}=-16.6$ (the completeness limit of the Croton catalog). Finally, we impose a maximum redshift of 0.17 , which ensures that we do not probe distances greater than $500 h^{-1} \mathrm{Mpc}$ (the size of the simulation cube). The resulting catalog contains roughly 300,000 galaxies, or about one thirtieth of the full Croton et al. (2006) sample.

There are two disadvantages to this simple approach. First, the vast majority of the galaxies in the simulation are discarded as a result of the imposed flux limits, despite the fact that they are sufficiently luminous to be of interest. Secondly, in order to assess projection effects, it would be useful to view galaxy associations from a variety of vantage points.

Fortunately, the periodic boundary conditions imposed on the simulations provide a way forward. Following part of the "random tiling" technique outlined by Blaizot et al. (2005), we generate a suite of mock redshift catalogs. Specifically, for each mock catalog, we begin by applying to the Millennium cube a translation in each of the three spatial directions, with the size of each translation being a random fraction of the box size $(500$ $\left.h^{-1} \mathrm{Mpc}\right)$. For any resulting coordinate value greater than the box size, we subtract the box size, thereby "wrapping around" the cube. Finally, we rotate the cube around each of the three spatial axes by a random (integer) multiple of $\pi / 2$. Using this approach, we create a suite of 30 mock redshift catalogs, sampling a total of about 9 million galaxies (comparable to the number of galaxies in the original data cube).

\subsection{Redshift-space Pair Statistics}

We then proceed to compute pair statistics on all of the mock redshift catalogs, selecting host and companion galaxies in the same manner outlined in Sections 3.1 and 3.2. One key difference in technique must be noted, however: given that the Millennium data is a redshift zero realization, we compute co-moving rather than physical projected separations in order to recover the correct $z=0$ physical separations.

Our results are presented in Figure 4 and in the first two columns of Table 4. Overall, the trends seen are broadly similar to those for SDSS shown in Figure 3. The distribution is again relatively flat for the smallest separation pairs, and increases towards the faint end as pair separation increases. This implies that the projected two point correlation becomes steeper on small scales as luminosity increases, which is consistent with the increase in steepness with stellar mass found for Millennium galaxies by Kitzbichler \& White (2008). For close pairs $\left(5<r_{p}<20 h^{-1} \mathrm{kpc}\right), N_{c}\left(M_{r}\right)$ peaks at a value of about 0.02 at $M_{r} \sim-20.75$. Given that $M^{*}$ is approximately -20.6 in the $r$ filter (Blanton et al. 2003a), this implies that $M^{*}$ galaxies are the most likely to have close companions. For close pairs over the range $-22<M_{r}<-18$, the mean $N_{c}$ is $0.0183 \pm 0.0001$, which is about $15 \%$ smaller than we found for SDSS. The most significant difference between SDSS and Millennium is seen for the larger separation pairs, in that the Millen- 
nium $N_{c}\left(M_{r}\right)$ is considerably steeper. We discuss a possible explanation for this in Section 6.1. However, given that we are primarily interested in the smallest separation pairs, the general agreement seen between SDSS and Millennium in this regime justifies further comparison between these samples.

\subsection{Projection Effects}

One of the most challenging aspects of close pair studies is the contamination of pair samples due to projection effects. For any observed galaxy pair, even when spectroscopic redshifts are available for both galaxies, one cannot be certain that the galaxies are close enough to merge. In addition, without measurements of transverse velocities, it is also possible that the relative velocities of the member galaxies are too high for coalescence to occur. For pairs with $5<r_{p}<20 h^{-1} \mathrm{kpc}$ and $\Delta v<500$ $\mathrm{km} \mathrm{s}^{-1}$, Patton et al. (2000) estimated this contamination to be on the order of $50 \%$. One must apply such a statistical correction for projection effects in order to relate measured pair statistics to their real-space (threedimensional) equivalents.

The three dimensional information available in the Croton et al. (2006) catalogs enables us to instead measure the level of contamination directly. For every pair of galaxies that we observe in our mock redshift catalogs, we can measure the three dimensional physical separation, along with the three dimensional relative velocity. If these quantities are less than $20 h^{-1} \mathrm{kpc}$ and $500 \mathrm{~km}$ $\mathrm{s}^{-1}$ respectively, it is likely that a merger is imminent (Patton et al. 2000). In addition, every pair satisfying these three dimensional criteria will also satisfy the redshift space criteria (i.e., in terms of $r_{p}$ and $\Delta v$ ) when viewed from any vantage point.

For our Millennium mock catalogs, we measure the fraction of close companions which satisfy the three dimensional criteria; following Patton et al. (2000), we refer to this fraction as $f_{3 D}$. Over the range $-22<M_{r}<$ -18 , we find an overall $f_{3 D}$ of $47.4 \% \pm 0.3 \%$. This compares well with the rough estimate of $f_{3 D} \sim 50 \%$ of Patton et al. (2000), although their estimate applies to different absolute magnitude criteria $\left(-21<M_{B}<-18\right.$ for both hosts and companions).

In addition to measuring the overall $f_{3 D}$, it is also useful to probe the dependence of this quantity on absolute magnitude. If it varies significantly with $M_{r}$, this will have implications for any merger rate estimates we wish to glean from our $N_{c}\left(M_{r}\right)$ measurements. The results are given in Figure 5, and in the third column of Table 4. Interestingly, $f_{3 D}$ is seen to vary by a factor of about three over the range in $M_{r}$ probed, decreasing from $\sim 56 \%$ at $M_{r} \lesssim M^{*}$ to about $17 \%$ at $M_{r} \sim-18$. This implies that most of the lowest luminosity close pairs in our Millennium mock catalogs are not candidates for imminent mergers, while at least half of the close pairs with $M_{r} \lesssim M^{*}$ are likely to undergo imminent mergers.

\section{CLOSE PAIR STATISTICS IN REAL SPACE}

\subsection{Millennium}

In the preceding section, we reported our measurement of $f_{3 D}\left(M_{r}\right)$ from the Millennium simulation. Multiplying this function by the redshift space close pair statistics reported in $\S 4.2$, we arrive at real space pair statistics, in which projection effects have been removed. In Figure6. we present these results for the Millennium simulation, along with the redshift-space results given earlier. We also provide tabulated values in column 4 of Table 4 , In real space, we find that $N_{c}\left(M_{r}\right)$ peaks at $M_{r} \sim M^{*}$, at a value of $\sim 0.011$, rather than $\sim 0.02$ in redshift space. Also, rather than being relatively flat (as it is in redshift space), we now see a strong decline towards fainter luminosities, as a result of the associated decline in $f_{3 D}$. The real space $N_{c}$ drops to about 0.002 at the faintest luminosities probed, which is much lower than in redshift space. These results imply that $L^{*}$ galaxies are much more likely to have close companions than $0.1 L^{*}$ galaxies. This striking difference is not apparent in redshift space, due to the presence of projection effects. We also see a hint that the real space $N_{c}$ declines at the bright end $\left(M_{r} \sim-23\right)$, though this is seen only in the most luminous bin in the simulations.

\section{2. $S D S S$}

The strong contribution from projection effects seen in the Millennium simulation has important implications for the SDSS redshift space pair statistics presented in $\S$ 3.4. In particular, the relatively flat $N_{c}\left(M_{r}\right)$ observed for SDSS close pairs almost certainly does not hold true in real space. Without three dimensional positions and velocities for SDSS galaxies, we are unable to directly remove projection effects. However, given the general agreement between the SDSS and Millennium redshift space pair statistics (see $\S$ 4.2), it seems reasonable to apply our knowledge of Millennium projection effects to our SDSS pair statistics. Multiplying the SDSS redshift space $N_{c}\left(M_{r}\right)$ by the Millennium $f_{3 D}\left(M_{r}\right)$ yields our best estimate of SDSS close pair statistics in real space. These results are given in Figure 6, as well as in Table 3. We find that the SDSS real space $N_{c}\left(M_{r}\right)$ is approximately 0.011 for $-22<M_{r}<-20$, and then drops to about 0.0055 for $-20<M_{r}<-18$. Given the larger error bars in our SDSS measurements, we cannot state with any certainty where the real-space $N_{c}\left(M_{r}\right)$ peaks; however, our results are consistent with a peak at $M_{r}<-20$, as seen in the Millennium simulations ( $(5.1)$.

\subsection{Comparison with Other Studies}

Most observed samples of close pairs have been too small to permit anything meaningful to be learned about the luminosity (or mass) dependence of close pair statistics. The most notable exception is $\mathrm{Xu}$ et al. (2004), who measure the pair fraction as a function of $K_{s}$-band absolute magnitude for galaxies in the 2MASS survey. While they generally probe higher luminosities $\left(-24.5<M_{K}<\right.$ -22.5 ) than we do, their two faintest bins (centered on $M_{k}=-22$ ) correspond roughly to our brightest SDSS bin $\left(M_{r}=-21.5\right)$; in this regime, they find a pair fraction of about $0.011 \pm 0.005$, which is consistent (within $1 \sigma$ ) of our $N_{c} \sim 0.019 \pm 0.002$ (Table 3), despite some differences between their pair criteria and ours. However, Xu et al. (2004) find that the pair fraction increases towards higher luminosities (albeit with large error bars), whereas we see a hinted of a decrease with luminosity in this regime with Millennium. On the other hand, our observed trend is consistent with the semi-analytic results of Khochfar \& Burkert (2001), who find that the $z=0$ 
merger fraction decreases as mass increases, over a range in masses which corresponds to $M_{r} \lesssim M^{*}$.

We also compare our results with those of Berrier et al. (2006), who measure $N_{c}$ using a combination of N-body simulations and semi-analytic models. They find that $N_{c}$ increases steadily as the cumulative co-moving number density increases. This is equivalent to an increase in $N_{c}$ towards fainter limiting $M_{r}$, over a range corresponding to $M_{r} \geq M_{r}^{*}$ (using the SDSS LF measurements of Blanton et al. (2003a)). We instead see a steady decrease in $N_{c}$ towards fainter $M_{r}$ over this range. However, Berrier et al. (2006) include all companions brighter than the given number density (or equivalently, $M_{r}$ ). With this approach, the cumulative number of close companions for a given galaxy can only go up as $M_{r}$ becomes fainter. It is therefore not surprising that their measurements of $N_{c}$ rise towards fainter $M_{r}$. Given that we require companions to be within a factor of two in luminosity of their host galaxy, there is no reason to expect agreement between our measurements of $N_{c}$ and those of Berrier et al. (2006). We note, however, that our definition of $N_{c}$ is likely to be a better tracer of the major merger rate.

\section{FROM CLOSE PAIR STATISTICS TO THE MERGER RATE}

\subsection{The Number Density of Close Companions}

We have measured the number of companions per galaxy $\left(N_{c}\right)$, which tells us which galaxies are most likely to have close companions. The real-space distributions seen in Figure 6 indicate that low luminosity galaxies are the least likely to have close companions, and presumably the least likely to undergo imminent mergers. However, low luminosity galaxies are much more common than luminous galaxies, and therefore it is still possible that most major mergers may occur between low luminosity galaxies.

We investigate this question by measuring the number of close companions per unit co-moving volume, hereafter $n_{c}$. In order to compute this quantity, we multiply the number of close companions per galaxy $\left(N_{c}\left(M_{r}\right)\right.$ by the co-moving number density of galaxies $\left(n\left(M_{r}\right)\right)$. For SDSS, we estimate $n\left(M_{r}\right)$ using the LF measurements of Blanton et al. (2003a), converting from the ${ }^{0.1} r$ filter to the $r$ filter using their recommended $M_{r}=M_{0.1_{r}}-0.16$. For Millennium, we measure the galaxy number density directly from the Croton et al. (2006) galaxy catalog. Like Li et al. (2007), we find that the Blanton et al. (2003a) LF matches the Croton et al. (2006) catalog quite well overall, though the simulations overpredict the number of galaxies at fainter luminosities (the excess is $\sim 50 \%$ at $\left.M_{r}=-18\right)$. We note that this excess helps to explain why $N_{c}\left(M_{r}\right)$ for wide separation pairs is steeper for Millennium than for SDSS (see $\S 4.2$ ).

The resulting measurements of $n_{c}\left(M_{r}\right)$ are given in Figure 7 and in Tables 3 and 4 . Excellent agreement is seen between SDSS and Millennium. When summed over the range $-22<M_{r}<-18$, the SDSS $n_{c}=$ $(2.10 \pm 0.13) \times 10^{-4} h^{3} \mathrm{Mpc}^{-3}$, which is consistent (within one sigma) with the Millennium $n_{c}=(1.98 \pm 0.02) \times$ $10^{-4} h^{3} \mathrm{Mpc}^{-3}$. In addition, the shapes of the distributions are very similar, with $n_{c} \sim 6 \times 10^{-5} h^{3} \mathrm{Mpc}^{-3} \mathrm{mag}^{-1}$ for $M_{r}>-21$, and a sharp drop in $n_{c}$ at higher lumi- nosities. For $M_{r}>-21$, it therefore appears that the decrease in the real-space $N_{c}\left(M_{r}\right)$ towards fainter absolute magnitudes is balanced by a corresponding increase in host galaxy number density. On the other hand, the relative scarcity of luminous host galaxies leads to the rapid decline in $n_{c}$ towards bright absolute magnitudes. From Figure 7, we conclude that at least $90 \%$ of all major mergers occur between galaxies which are fainter than $M_{r}=-21$. We note that, while there are no other directly comparable measurements in the literature, the shape of our observed $n_{c}\left(M_{r}\right)$ is qualitatively similar to the shape of the merging galaxy mass functions of Bundy et al. (2005) and Hopkins et al. (2006).

\subsection{The Galaxy Merger Rate}

We have reported estimates of the number of companions per galaxy $\left(N_{c}\right)$ and per unit volume $\left(n_{c}\right)$. With additional assumptions, both can be converted to merger rates. Following the formalism of Patton et al. (2000), we will refer to these as the galaxy merger rate (hereafter $\left.\mathcal{R}_{\mathrm{mg}}\right)$ and the volume merger rate $\left(\mathcal{R}_{\mathrm{mg}_{V}}\right)$ respectively. Assuming that two real-space close companions (ie., one galaxy pair) lead to one merger, and that the average timescale for such mergers is $T_{\mathrm{mg}}$, it follows that $\mathcal{R}_{\mathrm{mg}}=0.5 N_{c} / T_{\mathrm{mg}}$ and $\mathcal{R}_{\mathrm{mg}_{V}}=0.5 n_{c} / T_{\mathrm{mg}}$, where both $N_{c}$ and $n_{c}$ refer to real-space measurements (Figures 6 and (7).

To proceed further requires an estimate of $T_{\mathrm{mg}}$. We begin by taking the simplest approach, which is to assume that $T_{\mathrm{mg}}$ is equal to the dynamical friction timescale of a typical pair in the sample (after projection effects have been removed). We adopt the estimate of $0.5 \mathrm{Gyr}$ given by Patton et al. (2000), since our pairs are chosen with similar criteria. This timescale estimate is comparable to or somewhat larger than several more recent estimates given in the literature (van Dokkum 2005; Bell et al. 2006a; De Propris et al. 2007). While the merger timescale may depend on mass (and hence absolute magnitude), N-body simulations appear to indicate that mass ratio is the most significant factor (Jiang et al. 2008), and this should be independent of $M_{r}$ for the pair criteria we employ.

The resulting merger rates are plotted using the right hand axes in Figures [6] and [7. The galaxy merger rate is found to peak at about 0.01 mergers per galaxy per Gyr, while the volume merger rate plateaus at about $6 \times 10^{-5} h^{3} \mathrm{Mpc}^{-3} \mathrm{Gyr}^{-1} \mathrm{mag}^{-1}$. As the conversion from pair statistics $\left(N_{c}\right.$ and $\left.n_{c}\right)$ to merger rates $\left(\mathcal{R}_{\mathrm{mg}}\right.$ and $\mathcal{R}_{\mathrm{mg}_{V}}$ ) is independent of $M_{r}$, the trends with $M_{r}$ described in Sections 5 and 6.1 apply here as well. We again conclude that while galaxies brighter than $L^{*}$ have the highest likelihood of being involved in major mergers, most major mergers take place between galaxies fainter than $L^{*}$.

How do these merger rate estimates compare with others in the literature? De Propris et al. (2007) estimated the merger rate using two methods: close galaxy pairs (where both galaxies were required to have $-21<M_{B}<-18$ ) and high asymmetries (as an indicator of ongoing mergers). They found a merger fraction close to $2 \%$ for both methods, which corresponds to $\mathcal{R}_{\mathrm{mg}} \sim 0.02 \mathrm{Gyr}^{-1}$ using our $T_{\mathrm{mg}}=0.5$ Gyr. This is approximately twice as high as our result. 
De Propris et al. (2007) also report a volume merger rate of $(5.2 \pm 1.0) \times 10^{-4} h^{3} \mathrm{Mpc}^{-3} \mathrm{Gyr}^{-1}$, whereas we find $\mathcal{R}_{\mathrm{mg}_{V}}=(1.4 \pm 0.1) \times 10^{-4} h^{3} \mathrm{Mpc}^{-3} \mathrm{Gyr}^{-1}$ when summing over a comparable range in absolute magnitude $\left(-22<M_{r}<-19\right)$. After recomputing their result with our $T_{\mathrm{mg}}=0.5 \mathrm{Gyr}$ (instead of their $0.3 \mathrm{Gyr}$ ), we again find that their merger rate is roughly double ours. The most likely explanation for this difference is that both the pairs and asymmetry methods of De Propris et al. (2007) include both major and minor mergers, whereas our measurements apply to major merger candidates only.

Maller et al. (2006) measure the galaxy merger rate as a function of mass, mass ratio, and redshift, using a smoothed particle hydrodynamics (SPH) cosmological simulation. For mergers above a 1:2 mass ratio (comparable to our 1:2 luminosity ratio), they find the galaxy merger rate at $z=0.3$ to be $\mathcal{R}_{\mathrm{mg}}=0.054 \mathrm{Gyr}^{-1}$ for high mass galaxies. This range in mass corresponds approximately to $-18.8<M_{r}<-17.7$ (derived from Table 1 of Maller et al. (2006)). While they do not report precise values of $\mathcal{R}_{\mathrm{mg}}$ at lower redshifts, they do find that $\mathcal{R}_{\mathrm{mg}}$ declines quickly towards $z=0$, yielding $\mathcal{R}_{\mathrm{mg}} \lesssim 0.02 \mathrm{Gyr}^{-1}$ at $z<0.1$. This value is consistent with the $\mathcal{R}_{\mathrm{mg}} \sim 0.005 \mathrm{Gyr}^{-1}$ we find at $z \sim 0.03$ over this range in $M_{r}$, particularly given that no error bars on the Maller et al. (2006) estimate are available. Interestingly, they find that the merger rate is lower for lower mass galaxies, in agreement with the trends we find in Figure 6 (particularly for Millennium). Maller et al. (2006) also measure a volume merger rate of about $1 \times 10^{-4} h^{3} \mathrm{Mpc}^{-3} \mathrm{Gyr}^{-1}$ (with large error bars) for high mass galaxies at $z=0.1$; this is consistent with the $\mathcal{R}_{\mathrm{mg}_{V}} \sim 6 \times 10^{-5} h^{3} \mathrm{Mpc}^{-3} \mathrm{Gyr}^{-1}$ we find in Figure 7 In general, Maller et al. (2006) find that $\mathcal{R}_{\mathrm{mg}_{V}}$ declines towards lower masses (though this effect is significant only at $z \gtrsim 0.4$ ), implying that one might expect to see $\mathcal{R}_{\mathrm{mg}_{V}}$ decline at fainter absolute magnitudes than probed by our SDSS and Millennium catalogs.

Masjedi et al. (2006) measured the volume merger rate for pairs of luminous red galaxies (LRG's) in the SDSS, and found $\mathcal{R}_{\mathrm{mg}_{V}} \lesssim 0.6 \times 10^{4} \mathrm{Gyr}^{-1} \mathrm{Gpc}^{-3}$. Both members of their LRG's pairs were required to have absolute magnitudes of $-23.2<M_{g}<-21.2$, which is different in nature from our major merger requirement of $\left|\Delta M_{r}\right|<0.753$. While our SDSS pairs are less luminous than the Masiedi et al. (2006) LRG's, our Millennium volume merger rate estimates in Figure 7 yield a similar result $\left(\mathcal{R}_{\mathrm{mg}_{V}} \lesssim 1 \times 10^{4} \mathrm{Gyr}^{-1} h^{3} \mathrm{Mpc}^{-3}\right)$ in the LRG luminosity range. Finally, we note that while Masjedi et al. (2008) probe the luminosity dependence of close companions of LRG's in the SDSS, their (LRG) host galaxies are more luminous than the host galaxies we probe here, and most of their close companions are not luminous enough to qualify as major merger candidates; therefore, there is essentially no overlap between our studies. However, their Figure 3 is consistent with a major merger rate of $\mathcal{R}_{\mathrm{mg}} \sim 0.001 \mathrm{Gyr}^{-1}$ for typical LRG's, which is in qualitative agreement with the hinted drop of $N_{c}\left(M_{r}\right)$ towards high luminosities which we describe in $\oint 5.3$.

We caution the reader that our estimates of the merger rate depend on two quantities: the close pair statistics we have measured $\left(N_{c}\right.$ or $\left.n_{c}\right)$, and the merger timescale
$\left(T_{\mathrm{mg}}\right)$ that we have estimated. The latter is considerably less certain, and different choices of the merger timescale and its dependence on mass (or luminosity) may significantly change our estimated merger rates. The most useful check on our results comes from Kitzbichler \& White (2008), who use the Millennium simulation to devise fitting formulas for estimating the merger timescale for pairs within a given projected physical separation (hereafter $\left.T_{\text {merge }}\right)$. While none of their fitting formulas are directly applicable to our sample, the closest match comes from using their equation 9 with $r_{p}<30 h^{-1} \mathrm{kpc}$, $v_{p}<300 \mathrm{~km} \mathrm{~s}^{-1}$, and $z=0$. We approximate the limiting stellar mass as the median Croton et al. (2006) stellar mass of galaxies corresponding to the faintest allowable companions for a given host galaxy. We note also that the Kitzbichler \& White (2008) fitting formulas apply to pairs in which galaxy stellar masses differ by at most a factor of 4, whereas our sample consists of pairs in which galaxy luminosities differ by a most a factor of 2 .

These choices yield $T_{\text {merge }}$ estimates which increase monotonically from about 1 Gyr at $M_{r} \sim-23$ to 4.1 Gyr at $M_{r} \sim-17.5$ (the range covered by our Millennium sample). At face value, these results may appear to be at odds with the fixed merging timescale of $0.5 \mathrm{Gyr}$ that we adopted earlier in this section. However, there is an important difference: the Kitzbichler \& White (2008) timescale is relevant for projected pairs, while our timescale is for real space pairs. To make a direct comparison, our timescale of 0.5 Gyr needs to be divided by $f_{3 \mathrm{D}}\left(M_{r}\right)$; this leads to values of $T_{\text {merge }}$ ranging from 0.8 Gyr to 3.2 Gyr over the same range in $M_{r}$, which compare quite favorably with the preceding calculations. Therefore, despite significant mismatches between our sample and the Kitzbichler \& White (2008) fitting formula criteria, both approaches lead to a similar luminosity dependence of the merging timescales for projected pairs, and the overall timescales are comparable. Moreover, our estimates of $f_{3 \mathrm{D}}\left(M_{r}\right)$ (see Figure 5) provide additional insight into the nature of the increase in $T_{\text {merge }}$ with decreasing luminosity; namely, that this trend may be a consequence of most lower luminosity pairs having separations which are too large for merging to take place, rather than such systems simply undergoing a slower merging process. Nevertheless, it is clear that more work is needed in order to more accurately model the merging timescales of observed pair samples.

\subsection{The Remnant Fraction}

Given the above measurements of the merger rate, it is in principle possible to assess the merging history of low redshift galaxies. Following Patton et al. (2000), we estimate the fraction of galaxies which have undergone major mergers since $z=1$. This quantity, which is referred to as the remnant fraction $\left(f_{\text {rem }}\right)$, is given by

$$
f_{\mathrm{rem}}\left(M_{r}\right)=1-\prod_{j=1}^{N} \frac{1-N_{c}\left(M_{r}, z_{j}\right)}{1-0.5 N_{c}\left(M_{r}, z_{j}\right)},
$$

where $N_{c}\left(M_{r}\right)$ is measured in real space (e.g., $\S[5), z_{j}$ corresponds to a lookback time of $j T_{\mathrm{mg}}$, and $N$ refers to the number of merger timescales over the range $0<$ $z<1$ ( $N=15$ for our chosen cosmology). In principle, one needs to measure $N_{c}\left(M_{r}\right)$ at $0<z<1$ in order to 
accurately compute $f_{\text {rem }}\left(M_{r}\right)$. As we have only our low redshift $(z<0.1)$ measurements of $N_{c}\left(M_{r}\right)$ to work with, we make the simplifying assumption that $N_{c}\left(M_{r}\right)$ does not evolve with redshift. If instead $N_{c}\left(M_{r}\right)$ rises with redshift, as indicated by numerous studies (see $\S$ 1), the resulting remnant fractions will be higher (Patton et al. 2002). And of course, if the shape of $N_{c}\left(M_{r}\right)$ evolves strongly, the shape of our estimated $f_{\text {rem }}\left(M_{r}\right)$ will also be in error.

Nevertheless, we present our no-evolution estimate of the luminosity dependent remnant fraction in Figure 8 and in Tables 3 and 4 . The SDSS remnant fraction peaks at $9 \%$ for $M_{r}=-20.5$ (roughly $M^{*}$ ), and decreases to $4 \%$ over the range $-20<M_{r}<-18$. Similar trends are seen for Millennium, although the Millennium remnant fraction drops to $\sim 2 \%$ at $M_{r}=-18$.

\subsection{The Luminosity Density of Close Companions}

We demonstrated in Section 6.1 that our observed $n_{c}\left(M_{r}\right)$ implies that most major mergers occur between galaxies which are fainter than $L^{*}$. Of course, a major merger between two intrinsically faint galaxies affects much less stellar mass than a major merger of two luminous galaxies. Therefore, in terms of the overall luminosity density of stellar mass that is participating in mergers, the number density of close companions does not tell the full story. To address this question, we instead measure the luminosity of close companions per unit co-moving volume (hereafter $l_{c}\left(M_{r}\right)$ ), using luminosity as a proxy for stellar mass. We compute $l_{c}\left(M_{r}\right)$ as follows:

$$
l_{c}\left(M_{r}\right)=L_{c}\left(M_{r}\right) f_{3 \mathrm{~d}}\left(M_{r}\right) n\left(M_{r}\right),
$$

where $L_{c}\left(M_{r}\right)$ is the luminosity in close companions per galaxy (Patton et al. 2000) as measured in redshift space, $f_{3 d}\left(M_{r}\right)$ is as described in $\S 4.3$, and $n\left(M_{r}\right)$ is the co-moving number density of galaxies (see $\S 6.1$ ). When calculating luminosities, we use $M_{r}(\odot)=4.64$ (Yasuda et al. 2001).

The resulting measurements for both SDSS and Millennium are given in Figure 9 and in Tables 3 and 4. Excellent agreement is again seen between SDSS and Millennium. Both surveys exhibit a clear peak in $l_{c}\left(M_{r}\right)$ at (or very close to) $M^{*}$, with a peak value of $l_{c} \sim 8 \times 10^{5} L_{\odot} h^{5} \mathrm{Mpc}^{-3} \mathrm{mag}^{-1}$. The sharp decline towards the bright end is due to the lower number density of luminous host galaxies, while the decline towards the faint end is driven by the lower numbers and luminosities of companion galaxies. This distribution clearly implies that galaxies which have luminosities close to $L^{*}$ are the most relevant in terms of the overall involvement of stellar mass in major mergers.

\section{CONCLUSIONS}

We have measured the number of close companions per galaxy $\left(N_{c}\right)$ as a function of absolute magnitude for both the SDSS and the Millennium simulation. For SDSS, we construct samples of host and companion galaxies, and correct for spectroscopic incompleteness. For Millennium, we create a suite of mock redshift catalogs, averaging over different views of the Croton et al. (2006) cube. Using close pair criteria designed to identify imminent major mergers $\left(5<r_{p}<20 h^{-1} \mathrm{kpc}, \Delta v<500\right.$ $\mathrm{km} \mathrm{s}^{-1}$, and $\left.\left|\Delta M_{r}\right|<0.753\right)$, we find general agreement between the observations and simulations. In redshift space, $N_{c} \sim 0.02$ over the range $-22<M_{r}<-18$. The flatness of this distribution indicates that $N_{c}\left(M_{r}\right)$ does not simply trace the number density of galaxies; instead, small scale luminosity-dependent clustering appears to counteract this effect.

Using three dimensional positions and velocities available from the Millennium simulations, we measure the degree to which the detected galaxy pairs are contaminated by projection effects, and find that the contamination is a strong function of absolute magnitude, rising from $45 \%$ at $M_{r} \lesssim M^{*}$ to $\sim 83 \%$ at $M_{r}=-18$. We remove this contamination from both Millennium and SDSS pair statistics, yielding $N_{c}\left(M_{r}\right)$ measurements in real space. These measurements indicate that galaxies with $M_{r} \lesssim M^{*}$ are the most likely to be undergoing major mergers at low redshift. However, by computing the number density of close companions $\left(n_{c}\left(M_{r}\right)\right)$ in real space, we conclude that at least $90 \%$ of all major mergers occur between galaxies which are fainter than $M^{*}$. With additional assumptions, we also estimate the galaxy and volume merger rates, which trace the real-space $N_{c}\left(M_{r}\right)$ and $n_{c}\left(M_{r}\right)$ respectively. We estimate that at least $8 \%$ of $L^{*}$ galaxies are likely to have undergone a major merger since $z=1$, while this remnant fraction appears to be $\sim 4$ times smaller for $0.1 L^{*}$ galaxies. Finally, our measurements of the luminosity density of close companions indicate a clear peak at $M^{*}$, implying that $L^{*}$ galaxies dominate in terms of the overall amount of stellar mass involved in major mergers at low redshift.

Together, these results indicate that the low redshift merger rate depends strongly on luminosity (and presumably mass). This has a number of important implications. For example, one would not expect the merger rates of massive galaxies (e.g., Masjedi et al. (2006)) to agree with those of $L^{*}$ galaxies (e.g., De Propris et al. 2007). Also, the increase in projection effects for fainter galaxies indicates that samples of luminous galaxy pairs are more likely to provide bona fide merger candidates than samples of lower luminosity pairs. This is relevant if one wishes to assess the impact of merging on the constituent galaxies (e.g., enhanced star formation or disturbed morphologies). We also conclude that at low redshift, recent merging history is likely to be most important for galaxies which are relatively luminous. Finally, given the clear peak in $l_{c}\left(M_{r}\right)$, it appears that galaxies which are much more or much less luminous than $L^{*}$ are unlikely to play an important role in the overall evolution of galaxies via major mergers.

We thank the anonymous referee for a thoughtful and constructive report, which led to significant enhancements of this paper. We gratefully acknowledge the financial support of the Natural Sciences and Engineering Research Council (NSERC) of Canada, through a Discovery Grant to D. R. P. and a USRA to J. E. A.

Funding for the SDSS and SDSS-II has been provided by the Alfred P. Sloan Foundation, the Participating Institutions, the National Science Foundation, the U.S. Department of Energy, the National Aeronautics and Space Administration, the Japanese Monbukagakusho, the Max Planck Society, and the Higher Education Funding Council for England. The SDSS Web Site 
is http://www.sdss.org/

The SDSS is managed by the Astrophysical Research Consortium for the Participating Institutions. The Participating Institutions are the American Museum of Natural History, Astrophysical Institute Potsdam, University of Basel, University of Cambridge, Case Western Reserve University, University of Chicago, Drexel University, Fermilab, the Institute for Advanced Study, the Japan Participation Group, Johns Hopkins University, the Joint Institute for Nuclear Astrophysics, the Kavli Institute for Particle Astrophysics and Cosmology, the Korean Scientist Group, the Chinese Academy of Sciences (LAMOST), Los Alamos National Laboratory, the
Max-Planck-Institute for Astronomy (MPIA), the MaxPlanck-Institute for Astrophysics (MPA), New Mexico State University, Ohio State University, University of Pittsburgh, University of Portsmouth, Princeton University, the United States Naval Observatory, and the University of Washington.

The Millennium Run simulation used in this paper was carried out by the Virgo Supercomputing Consortium at the Computing Centre of the Max-Planck Society in Garching. The semianalytic galaxy catalogue is publicly available at http://www.mpa-garching.mpg.de/galform/agnpaper.

\section{REFERENCES}

Abazajian, K., et al. 2003, AJ, 126, 2081

Adelman-McCarthy, J. K., et al. 2007, ApJS, 172, 634

Allam, S. S., Tucker, D. L., Smith, J. A., Lee, B. C., Annis, J., Lin, H., Karachentsev, I. D., \& Laubscher, B. E. 2004, AJ, 127, 1883

Alonso, M. S., Tissera, P. B., Coldwell, G., \& Lambas, D. G. 2004, MNRAS, 352, 1081

Barton, E. J., Arnold, J. A., Zentner, A. R., Bullock, J. S., \& Wechsler, R. H. 2007, ApJ, 671, 1538

Bell, E. F., Phleps, S., Somerville, R. S., Wolf, C., Borch, A., \& Meisenheimer, K. 2006a, ApJ, 652, 270

Bell, E. F., et al. 2006b, ApJ, 640, 241

Berrier, J. C., Bullock, J. S., Barton, E. J., Guenther, H. D., Zentner, A. R., \& Wechsler, R. H. 2006, ApJ, 652, 56

Binney, J., and Tremaine, S. 1987, in Galactic Dynamics (Princeton: Princeton University Press)

Blaizot, J., Wadadekar, Y., Guiderdoni, B., Colombi, S. T. Bertin, E., Bouchet, F. R., Devriendt, J. E. G., \& Hatton, S. 2005, MNRAS, 360, 159

Blanton, M. R., et al. 2003a, ApJ, 592, 819

Blanton, M. R., Lin, H., Lupton, R. H., Maley, F. M., Young, N., Zehavi, I., \& Loveday, J. 2003b, AJ, 125, 2276

Blanton, M. R., \& Roweis, S. 2007, AJ, 133, 734

Brough, S., Forbes, D. A., Kilborn, V. A., \& Couch, W. 2006, MNRAS, 370, 1223

Brown, M. J. I., Dey, A., Jannuzi, B. T., Brand, K., Benson, A. J., Brodwin, M., Croton, D. J., \& Eisenhardt, P. R. 2007, ApJ, 654, 858

Bundy, K., Fukugita, M., Ellis, R. S., Kodama, T., \& Conselice, C. J. 2004, ApJ, 601, L123

Bundy, K., Ellis, R. S., \& Conselice, C. J. 2005, ApJ, 625, 621

Bundy, K., Treu, T., \& Ellis, R. S. 2007, ApJ, 665, L5

Carlberg, R. G., et al. 2000, ApJ, 532, L1

Conselice, C. J., Bershady, M. A., Dickinson, M., \& Papovich, C. 2003, AJ, 126, 1183

Conselice, C. J., Rajgor, S., \& Myers, R. 2008, MNRAS, 386, 909

Cox, T. J., Jonsson, P., Primack, J. R., \& Somerville, R. S. 2006, MNRAS, 373, 1013

Cox, T. J., Jonsson, P., Somerville, R. S., Primack, J. R., \& Dekel, A. 2008, MNRAS, 384, 386

Coziol, R., \& Plauchu-Frayn, I. 2007, AJ, 133, 2630

Croton, D. J., et al. 2006, MNRAS, 365, 11

Dasyra, K. M., et al. 2006a, ApJ, 638, 745

Dasyra, K. M., et al. 2006b, ApJ, 651, 835

De Propris, R., Liske, J., Driver, S. P., Allen, P. D., \& Cross, N. J. G. 2005, AJ, 130, 1516

De Propris, R., Conselice, C. J., Liske, J., Driver, S. P., Patton, D. R., Graham, A. W., \& Allen, P. D. 2007, ApJ, 666, 212

Eisenstein, D. J., et al. 2001, AJ, 122, 2267

Ellison, S. L., Patton, D. R., Simard, L., \& McConnachie, A. W. 2008, AJ, 135, 1877

Geller, M. J., Kenyon, S. J., Barton, E. J., Jarrett, T. H., \& Kewley, L. J. 2006, AJ, 132, 2243

Goto, T. 2005, MNRAS, 357, 937

Guo, Q., \& White, S. D. M. 2008, MNRAS, 384, 2

Hernández-Toledo, H. M., Avila-Reese, V., Conselice, C. J., \& Puerari, I. 2005, AJ, 129, 682
Hopkins, P. F., Somerville, R. S., Hernquist, L., Cox, T. J., Robertson, B., \& Li, Y. 2006, ApJ, 652, 864

Hsieh, B. C., Yee, H. K. C., Lin, H., Gladders, M. D., \& Gilbank, D. G. 2008, ArXiv e-prints, 804, arXiv:0804.1604

Jiang, C. Y., Jing, Y. P., Faltenbacher, A., Lin, W. P., \& Li, C. 2008, ApJ, 675, 1095

Kampczyk, P., et al. 2007, ApJS, 172, 329

Kartaltepe, J. S., et al. 2007, ApJS, 172, 320

Kewley, L. J., Geller, M. J., \& Barton, E. J. 2006, AJ, 131, 2004

Khochfar, S., \& Burkert, A. 2001, ApJ, 561, 517

Kitzbichler, M. G., \& White, S. D. M. 2008, ArXiv e-prints, 804, arXiv:0804.1965

Lambas, D. G., Tissera, P. B., Alonso, M. S., \& Coldwell, G. 2003, MNRAS, 346, 1189

Le Fèvre, O., et al. 2000, MNRAS, 311, 565

Li, C., Kauffmann, G., Jing, Y. P., White, S. D. M., Börner, G., \& Cheng, F. Z. 2006, MNRAS, 368, 21

Li, C., Jing, Y. P., Kauffmann, G., Börner, G., Kang, X., \& Wang, L. 2007, MNRAS, 376, 984

Li, C., Kauffmann, G., Heckman, T. M., Jing, Y. P., \& White, S. D. M. 2008, MNRAS, 385, 1903

Lin, H., Yee, H. K. C., Carlberg, R. G., Morris, S. L., Sawicki, M., Patton, D. R., Wirth, G., \& Shepherd, C. W. 1999, ApJ, 518,533

Lin, L., et al. 2004, ApJ, 617, L9

Lin, L., et al. 2008, ArXiv e-prints, 802, arXiv:0802.3004

Lotz, J. M., et al. 2008, ApJ, 672, 177

Maller, A. H., Katz, N., Kereš, D., Davé, R., \& Weinberg, D. H. 2006, ApJ, 647, 763

Masjedi, M., et al. 2006, ApJ, 644, 54

Masjedi, M., Hogg, D. W., \& Blanton, M. R. 2008, ApJ, 679, 260

Miles, T. A., Raychaudhury, S., \& Russell, P. A. 2006, MNRAS, 373,1461

Moss, C. 2006, MNRAS, 373, 167

Naab, T., Khochfar, S., \& Burkert, A. 2006, ApJ, 636, L81

Nikolic, B., Cullen, H., \& Alexander, P. 2004, MNRAS, 355, 874

Nolan, L. A., Raychaudhury, S., \& Kabán, A. 2007, MNRAS, 375, 381

Norberg, P., et al. 2001, MNRAS, 328, 64

Norberg, P., et al. 2002, MNRAS, 332, 827

Owers, M. S., Blake, C., Couch, W. J., Pracy, M. B., \& Bekki, K. 2007, MNRAS, 833

Patton, D. R., Carlberg, R. G., Marzke, R. O., Pritchet, C. J., da Costa, L. N. \& Pellegrini, P. S. 2000, ApJ, 536, 153

Patton, D. R., et al. 2002, ApJ, 565, 208

Patton, D. R., Grant, J. K., Simard, L., Pritchet, C. J., Carlberg, R. G., \& Borne, K. D. 2005, AJ, 130, 2043

Rawat, A., Hammer, F., Kembhavi, A. K., \& Flores, H. 2008, ArXiv e-prints, 804, arXiv:0804.0078

Robotham, A., Wallace, C., Phillipps, S., \& De Propris, R. 2006, ApJ, 652, 1077

Ryan, R. E., Jr., Cohen, S. H., Windhorst, R. A., \& Silk, J. 2008, ApJ, 678, 751

Scarlata, C., et al. 2007, ApJS, 172, 494

Smith, B. J., Struck, C., Hancock, M., Appleton, P. N.,

Charmandaris, V., \& Reach, W. T. 2007, AJ, 133, 791

Sol Alonso, M., Lambas, D. G., Tissera, P., \& Coldwell, G. 2006, MNRAS, 367, 1029 
Springel, V., et al. 2005, Nature, 435, 629

Strauss, M. A., et al. 2002, AJ, 124, 1810

Tegmark, M., et al. 2004, ApJ, 606, 702

Tran, K.-V. H., van Dokkum, P., Franx, M., Illingworth, G. D., Kelson, D. D., \& Schreiber, N. M. F. 2005, ApJ, 627, L25

van Dokkum, P. G., Franx, M., Fabricant, D., Kelson, D. D., \& Illingworth, G. D. 1999, ApJ, 520, L95

van Dokkum, P. G. 2005, AJ, 130, 2647

Wang, J. L., Xia, X. Y., Mao, S., Cao, C., Wu, H., \& Deng, Z. G. 2006, ApJ, 649, 722
Woods, D. F., Geller, M. J., \& Barton, E. J. 2006, AJ, 132, 197

Woods, D. F., \& Geller, M. J. 2007, AJ, 134, 527

Xu, C. K., Sun, Y. C., \& He, X. T. 2004, ApJ, 603, L73

Yasuda, N., et al. 2001, AJ, 122, 1104

York, D. G., et al. 2000, AJ, 120, 1579

Zandivarez, A., Martínez, H. J., \& Merchán, M. E. 2006, ApJ, 650,137

Zehavi, I., et al. 2002, ApJ, 571, 172 
TABLE 1

SDSS Host Galaxies

\begin{tabular}{cccccc}
\hline Host ObjID & RA & Dec & $z$ & $M_{r}$ & Companion ObjID \\
\hline 587724198275842071 & 10.610426 & 14.70509 & 0.05539 & -19.72 & 587724198275842073 \\
587724197207670886 & 23.645067 & 13.18706 & 0.06304 & -20.17 & 587724197207670885 \\
587724197207670885 & 23.646278 & 13.18500 & 0.06226 & -20.50 & 587724197207670886 \\
587731513153159200 & 42.731424 & 0.36690 & 0.04384 & -20.10 & 587731513153159199 \\
587731513153159199 & 42.739416 & 0.36940 & 0.04423 & -19.76 & 587731513153159200 \\
\hline
\end{tabular}

TABLE 2

SDSS Redshift Space $N_{c}\left(M_{r}\right)$ as a Function of Projected Pair Separation

\begin{tabular}{ccccc}
\hline$r_{p}$ range & $-22<M_{r}<-21$ & $-21<M_{r}<-20$ & $-20<M_{r}<-19$ & $-19<M_{r}<-18$ \\
$\left(h^{-1} \mathrm{kpc}\right)$ & $(\bar{z}=0.107)$ & $(\bar{z}=0.074)$ & $(\bar{z}=0.048)$ & $(\bar{z}=0.031)$ \\
\hline $5-20$ & $0.0187 \pm 0.0018$ & $0.0238 \pm 0.0016$ & $0.0172 \pm 0.0018$ & $0.0226 \pm 0.0034$ \\
$20-40$ & $0.0225 \pm 0.0018$ & $0.0275 \pm 0.0016$ & $0.0298 \pm 0.0020$ & $0.0284 \pm 0.0026$ \\
$40-60$ & $0.0174 \pm 0.0015$ & $0.0332 \pm 0.0015$ & $0.0345 \pm 0.0016$ & $0.0329 \pm 0.0024$ \\
$60-80$ & $0.0180 \pm 0.0013$ & $0.0346 \pm 0.0011$ & $0.0389 \pm 0.0016$ & $0.0398 \pm 0.0026$ \\
$80-100$ & $0.0210 \pm 0.0011$ & $0.0360 \pm 0.0011$ & $0.0405 \pm 0.0016$ & $0.0470 \pm 0.0028$ \\
\hline
\end{tabular}

TABLE 3

SDSS Close Pair Statistics

\begin{tabular}{ccccccc}
\hline$M_{r}$ & $\begin{array}{c}N_{c} \\
\text { (redshift-space) }\end{array}$ & $\begin{array}{c}f_{3 D} \\
(\%)\end{array}$ & $\begin{array}{c}N_{c} \\
(\text { real-space) }\end{array}$ & $n_{c}$ & $\begin{array}{c}f_{\text {rem }} \\
(\%)\end{array}$ & $l_{c}$ \\
\hline-21.50 & $0.0187 \pm 0.0018$ & $55.3 \pm 0.4$ & $0.0103 \pm 0.0010$ & $1.52 \pm 0.15$ & $7.5 \pm 0.7$ & $3.44 \pm 0.37$ \\
-20.50 & $0.0238 \pm 0.0016$ & $52.5 \pm 0.3$ & $0.0125 \pm 0.0008$ & $6.93 \pm 0.44$ & $9.0 \pm 0.6$ & $7.97 \pm 0.58$ \\
-19.50 & $0.0172 \pm 0.0018$ & $33.8 \pm 0.6$ & $0.0058 \pm 0.0006$ & $5.78 \pm 0.60$ & $4.3 \pm 0.4$ & $2.81 \pm 0.33$ \\
-18.50 & $0.0226 \pm 0.0034$ & $22.8 \pm 0.7$ & $0.0052 \pm 0.0008$ & $6.78 \pm 1.04$ & $3.8 \pm 0.6$ & $1.57 \pm 0.26$ \\
\hline
\end{tabular}

TABLE 4

Millennium Close Pair Statistics

\begin{tabular}{ccccccc}
\hline$M_{r}$ & $\begin{array}{c}N_{c} \\
\text { (redshift-space) }\end{array}$ & $\begin{array}{c}f_{3 D} \\
(\%)\end{array}$ & $\begin{array}{c}N_{c} \\
\text { (real-space) }\end{array}$ & $n_{c}$ & $\begin{array}{c}f_{\text {rem }} \\
(\%)\end{array}$ & $l_{c}$ \\
\hline-22.75 & $0.0142 \pm 0.0010$ & $45.9 \pm 3.0$ & $0.0068 \pm 0.0007$ & $0.03 \pm 0.00$ & $5.0 \pm 0.5$ & 0.170 .01 \\
-22.25 & $0.0173 \pm 0.0004$ & $57.9 \pm 1.2$ & $0.0101 \pm 0.0003$ & $0.18 \pm 0.01$ & $7.3 \pm 0.2$ & 0.790 .02 \\
-21.75 & $0.0163 \pm 0.0002$ & $54.9 \pm 0.5$ & $0.0089 \pm 0.0001$ & $0.71 \pm 0.01$ & $6.5 \pm 0.1$ & 2.050 .02 \\
-21.25 & $0.0185 \pm 0.0002$ & $55.5 \pm 0.6$ & $0.0103 \pm 0.0001$ & $2.52 \pm 0.03$ & $7.5 \pm 0.1$ & 5.020 .06 \\
-20.75 & $0.0207 \pm 0.0002$ & $55.5 \pm 0.4$ & $0.0115 \pm 0.0001$ & $5.57 \pm 0.06$ & $8.3 \pm 0.1$ & 7.760 .07 \\
-20.25 & $0.0191 \pm 0.0002$ & $48.5 \pm 0.6$ & $0.0093 \pm 0.0002$ & $6.85 \pm 0.12$ & $6.8 \pm 0.1$ & 6.580 .07 \\
-19.75 & $0.0176 \pm 0.0002$ & $36.6 \pm 0.8$ & $0.0065 \pm 0.0002$ & $6.39 \pm 0.18$ & $4.7 \pm 0.1$ & 3.950 .06 \\
-19.25 & $0.0157 \pm 0.0003$ & $29.4 \pm 0.7$ & $0.0046 \pm 0.0001$ & $6.03 \pm 0.16$ & $3.4 \pm 0.1$ & 2.380 .04 \\
-18.75 & $0.0162 \pm 0.0004$ & $25.0 \pm 0.9$ & $0.0040 \pm 0.0001$ & $6.09 \pm 0.19$ & $3.0 \pm 0.1$ & 1.520 .04 \\
-18.25 & $0.0159 \pm 0.0006$ & $18.6 \pm 1.2$ & $0.0029 \pm 0.0002$ & $5.44 \pm 0.35$ & $2.2 \pm 0.1$ & 0.860 .04 \\
-17.75 & $0.0149 \pm 0.0009$ & $15.4 \pm 1.9$ & $0.0023 \pm 0.0003$ & $5.41 \pm 0.71$ & $1.7 \pm 0.2$ & 0.560 .04 \\
\hline
\end{tabular}




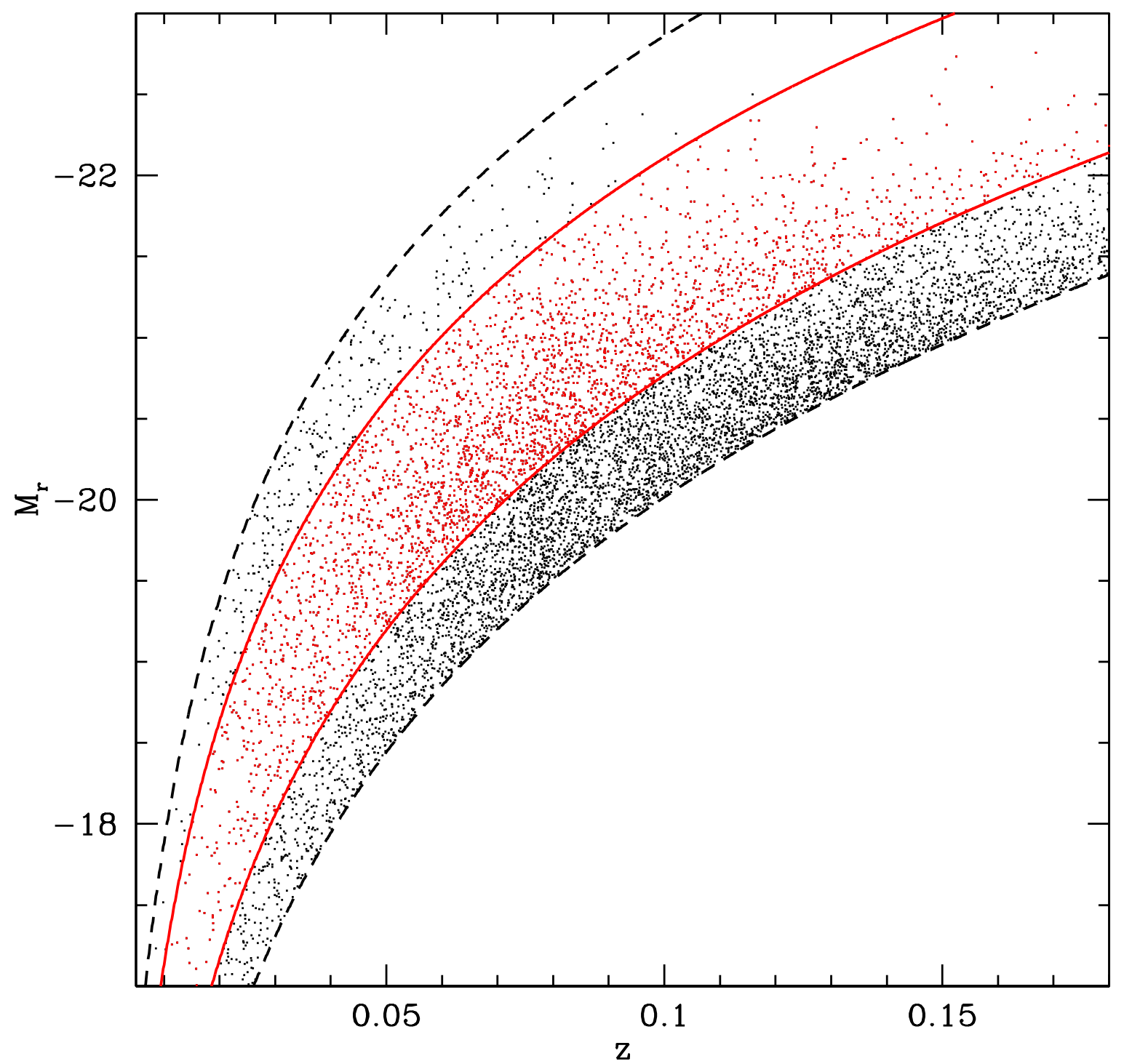

FIG. 1.- Absolute magnitude is plotted versus redshift for 10,000 galaxies selected at random from our SDSS spectroscopic catalog of about 337,000 galaxies. The dashed (black) lines enclose all potential companion galaxies; galaxies within this region have $14.5<m_{r}<17.5$. The solid (red) lines enclose all potential host galaxies; galaxies within this region differ by at least 0.753 magnitudes from the dashed lines. For these potential host galaxies, all companions within a luminosity ratio of 1:2 are therefore detectable. 

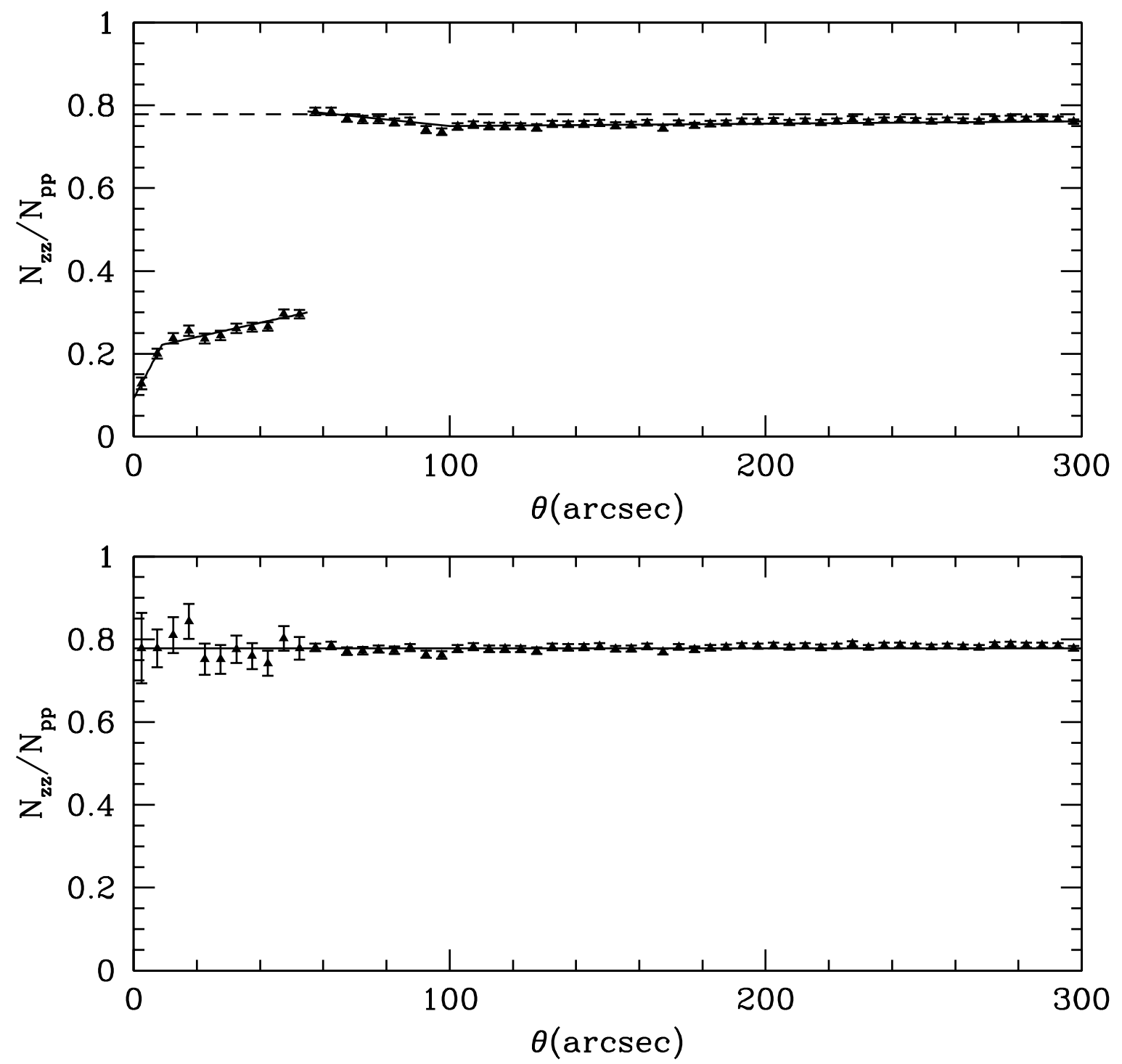

FIG. 2.- The ratio of spectroscopic to photometric pairs $\left(N_{z z} / N_{p p}\right)$ is plotted versus angular separation $(\theta)$, with error bars computed using the Jackknife technique. (a) The upper panel shows the data before corrective weights have been applied, and a substantial deficit of spectroscopic pairs is seen at small separations $\left(\theta<55^{\prime \prime}\right)$. At larger separations, $N_{z z} / N_{p p}$ converges to the square of the overall spectroscopic completeness of the sample $\left(f_{s}^{2} \sim 0.78\right)$, as indicated by the dashed line. The solid lines show our model fit to the data, which is used to correct for the small scale spectroscopic incompleteness. (b) The lower panel shows the data after these weights have been applied. The corrected data points provide an excellent fit to the overall spectroscopic completeness (solid line), confirming that the small scale incompleteness has been successfully removed. 


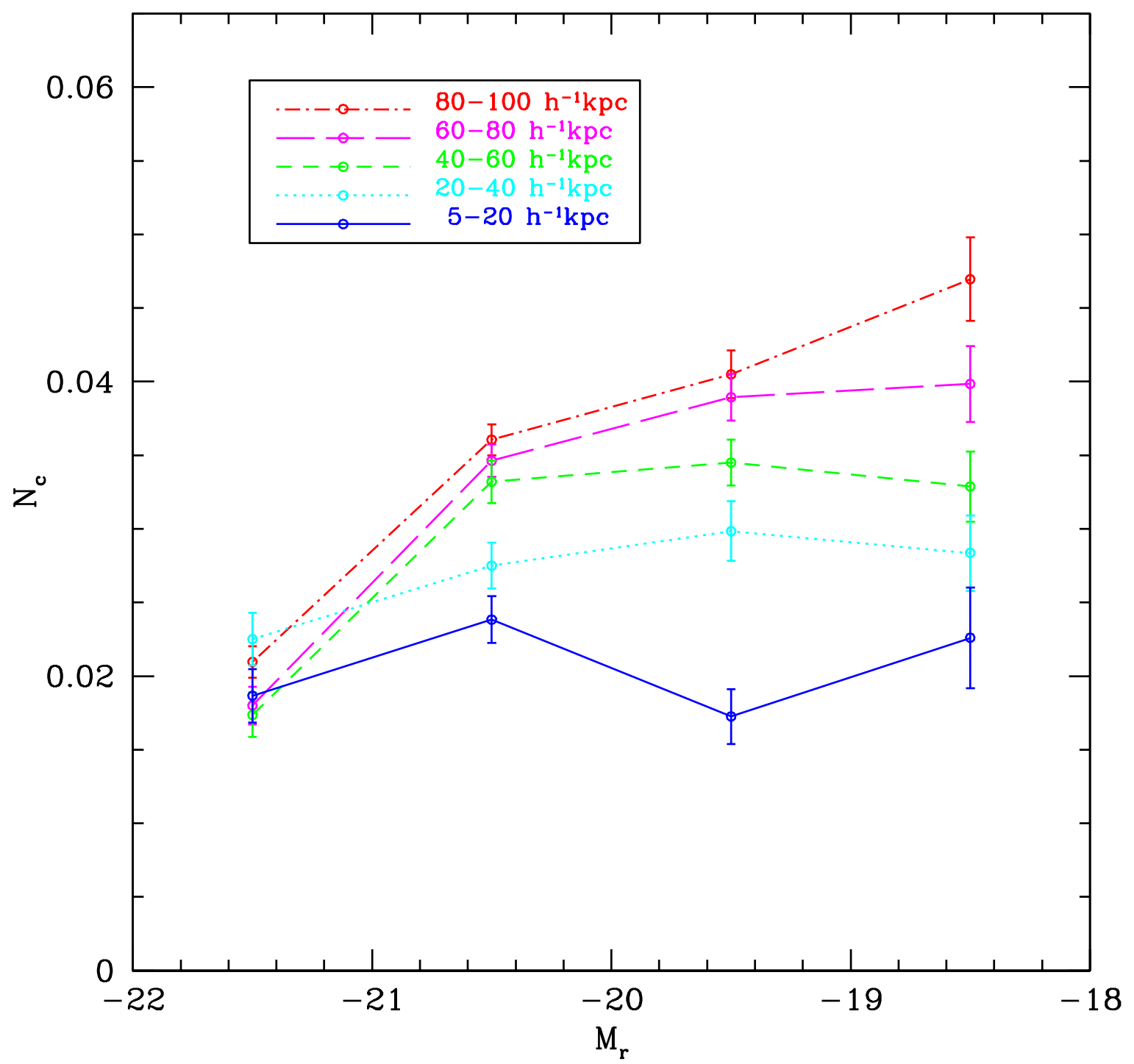

FIG. 3. $-N_{c}$ is plotted versus absolute magnitude for SDSS, for pairs in five ranges of projected separation. Over the range $-22<M_{r}<$ -18 , the mean $N_{c}$ for close pairs $\left(5-20 h^{-1} \mathrm{kpc}\right)$ is $0.021 \pm 0.001$. The $N_{c}$ distribution is approximately flat for the smallest separation pairs, and becomes steeper as pair separation increases. 


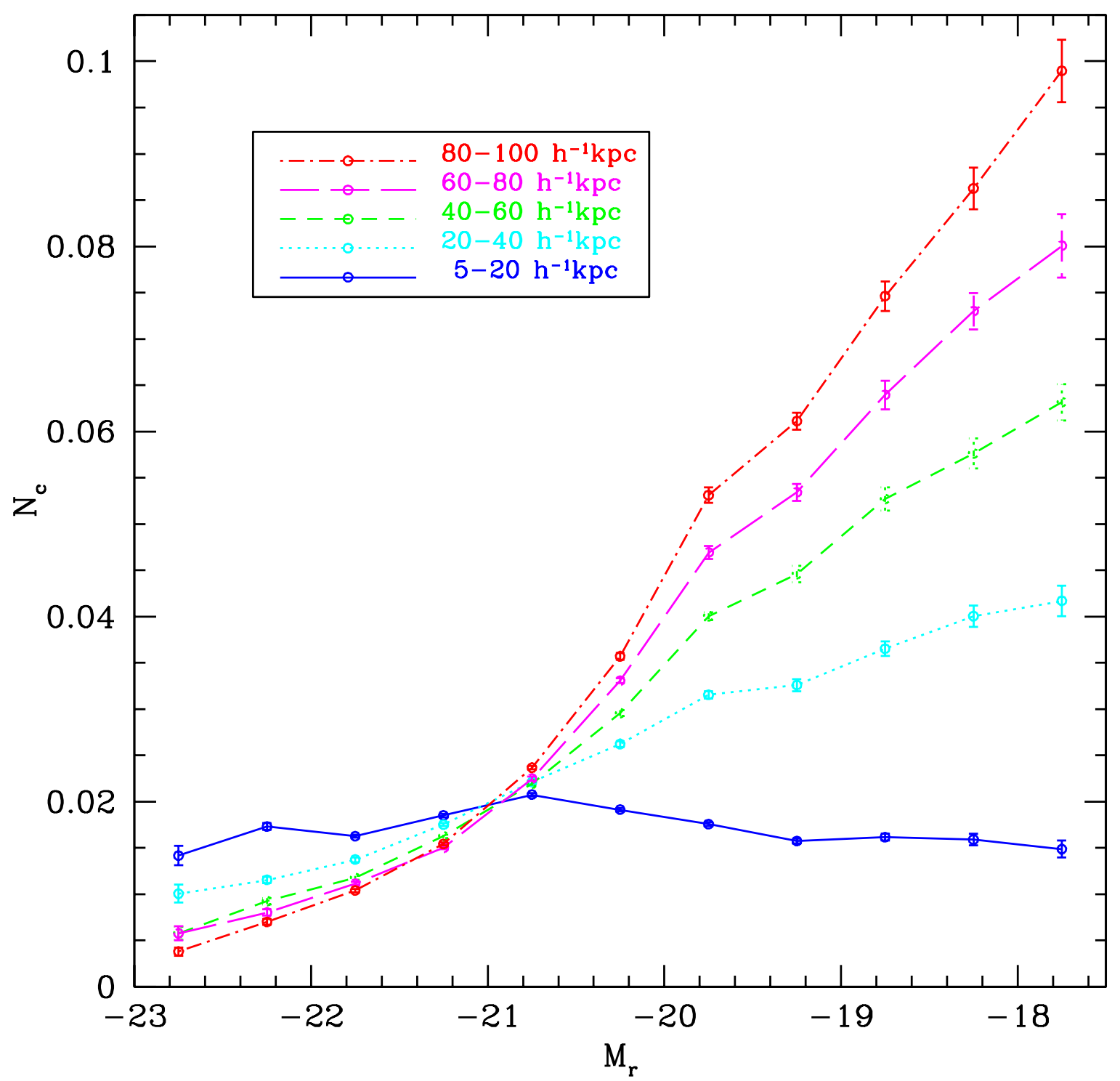

FIG. 4. $-N_{c}$ is plotted versus absolute magnitude for the Millennium simulation, for pairs in five ranges of projected separation. As with SDSS, the $N_{c}$ distribution is found to be approximately flat for the smallest pair separations. Over the range $-22<M_{r}<-18$, the mean $N_{c}$ for close pairs is $0.0183 \pm 0.0001$, which is about $15 \%$ lower than found for SDSS. The Millennium $N_{c}$ distribution steepens towards the faint end as pair separation increases. This is similar to what was seen for SDSS, but is considerably steeper. 


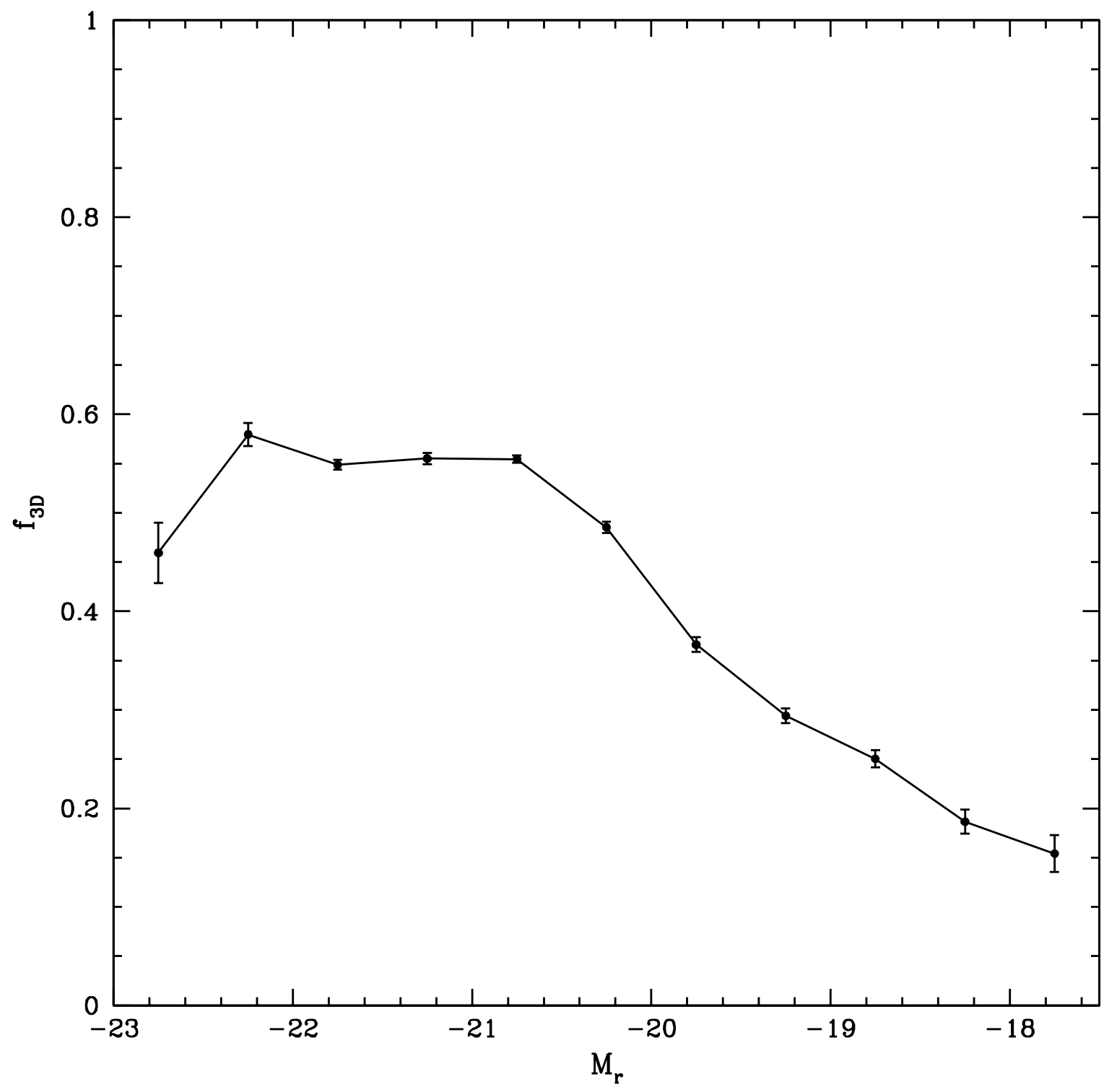

FIG. 5.- Using the Millennium simulation, the fraction of companions which are close in three dimensions $\left(f_{3 d}\right)$ is plotted versus absolute magnitude for close pairs $\left(5-20 h^{-1} \mathrm{kpc}\right)$. Projection effects are seen to increase towards fainter absolute magnitudes. 


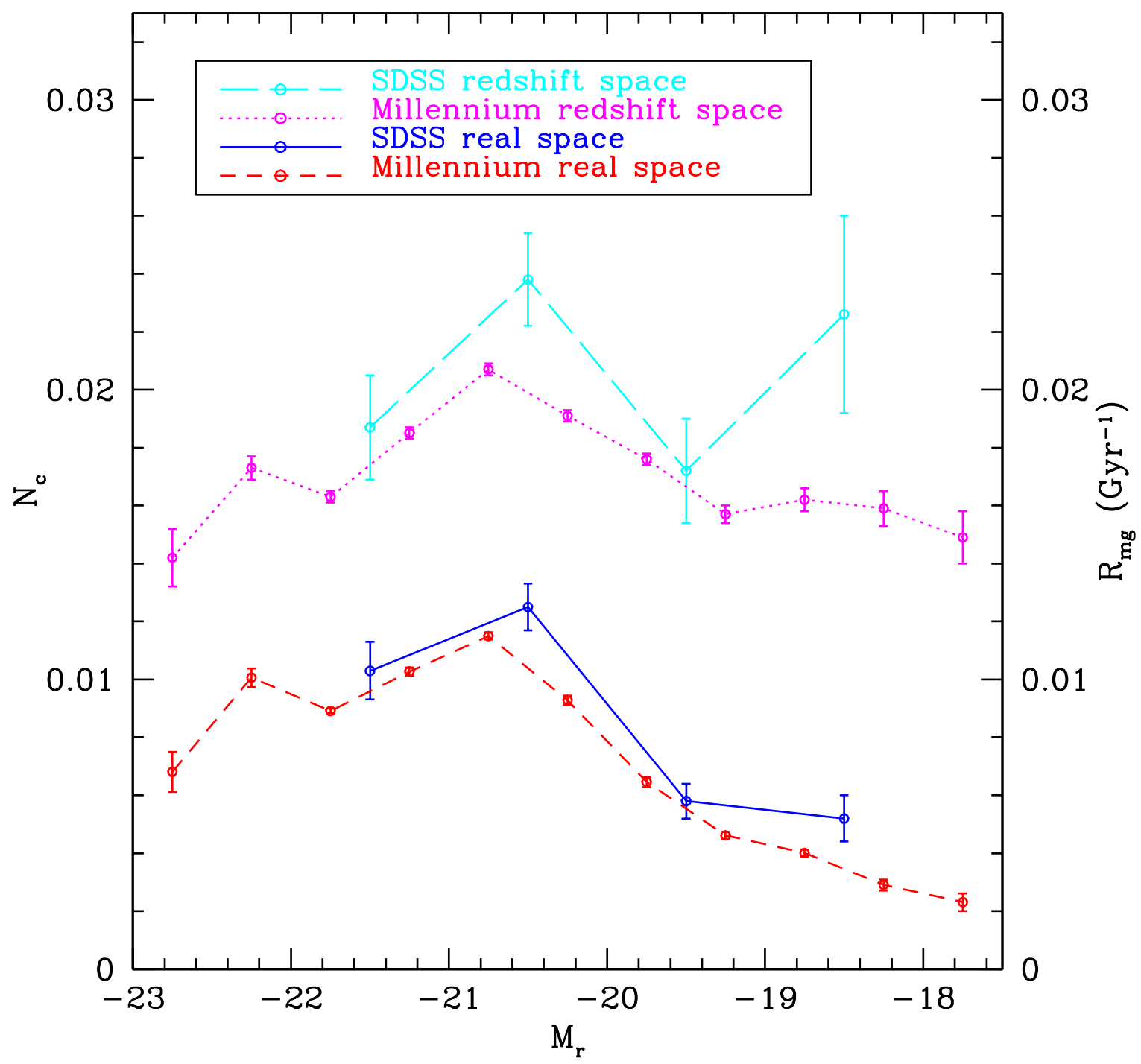

FIG. 6. - $N_{c}\left(M_{r}\right)$ is plotted in both redshift space and real space, for close pairs $\left(5-20 h^{-1} \mathrm{kpc}\right)$. For both SDSS and Millennium, conversion from redshift space to real space pair statistics (ie., the removal of projection effects) was carried out by multiplying $N_{c}\left(M_{r}\right)$ by the Millennium $f_{3 D}\left(M_{r}\right)$. The right hand axis displays the corresponding scale for the galaxy merger rate $\left(\mathcal{R}_{\mathrm{mg}}\right)$, and is relevant only for measurements in real space. 


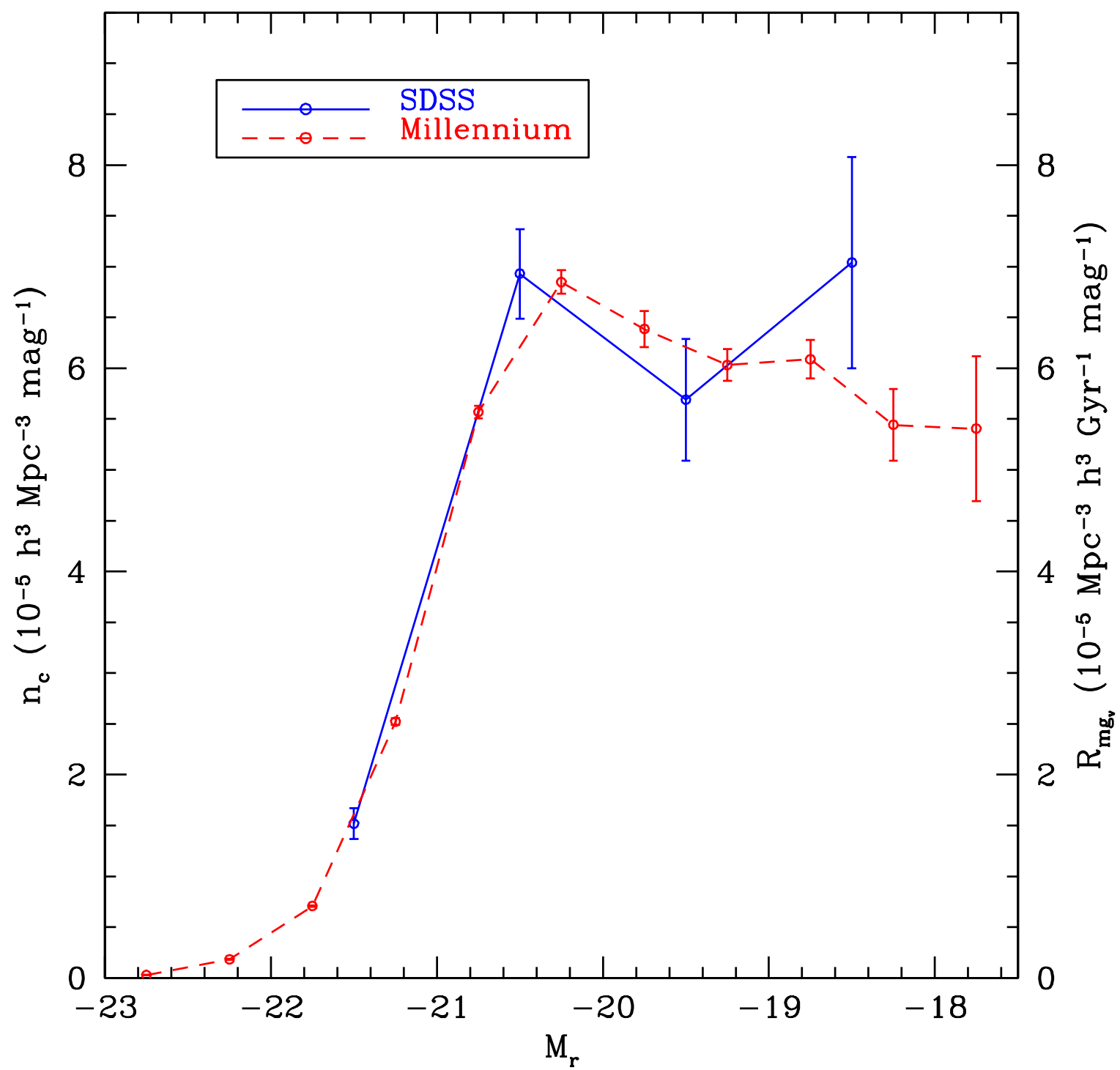

FIG. 7. - The number of close companions per unit co-moving volume $\left(n_{c}\right)$ is plotted versus $M_{r}$ for SDSS and for Millennium. In both cases, $n_{c}$ is computed by multiplying the real-space $N_{c}$ by the number density of galaxies. Excellent agreement is seen between the two samples. We conclude that most mergers occur between galaxies with $M_{r}>-21$. The right hand axis displays the corresponding volume merger rate. 


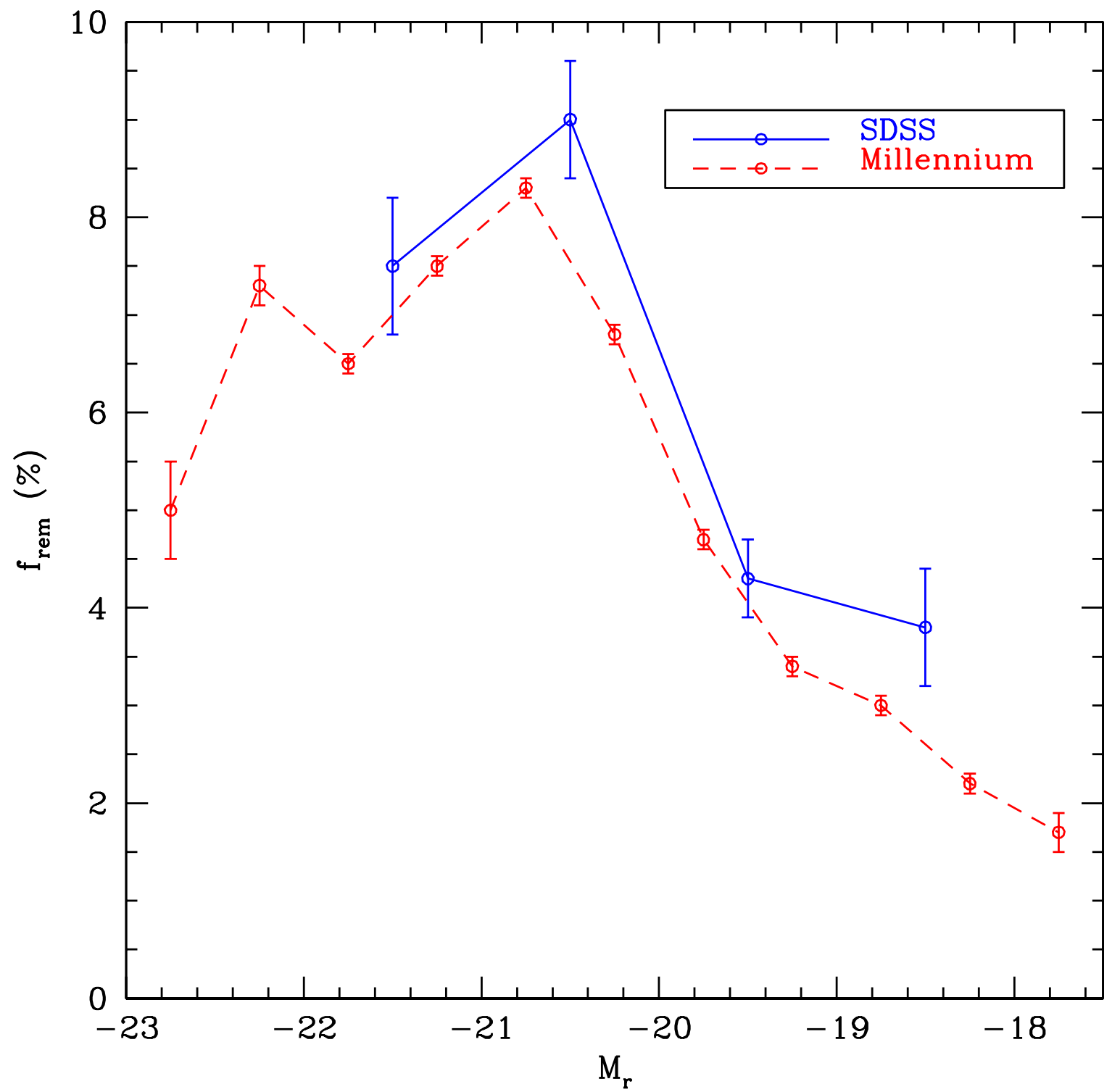

FIG. 8. - The remnant fraction $f_{\text {rem }}$, expressed as a percentage, is plotted versus $M_{r}$ for SDSS and for Millennium. In both cases, the error bars are computed using only the uncertainties in $N_{c}$. Given the assumptions that go into the calculation of $f_{\text {rem }}$, the true uncertainties are likely to be considerably larger. 


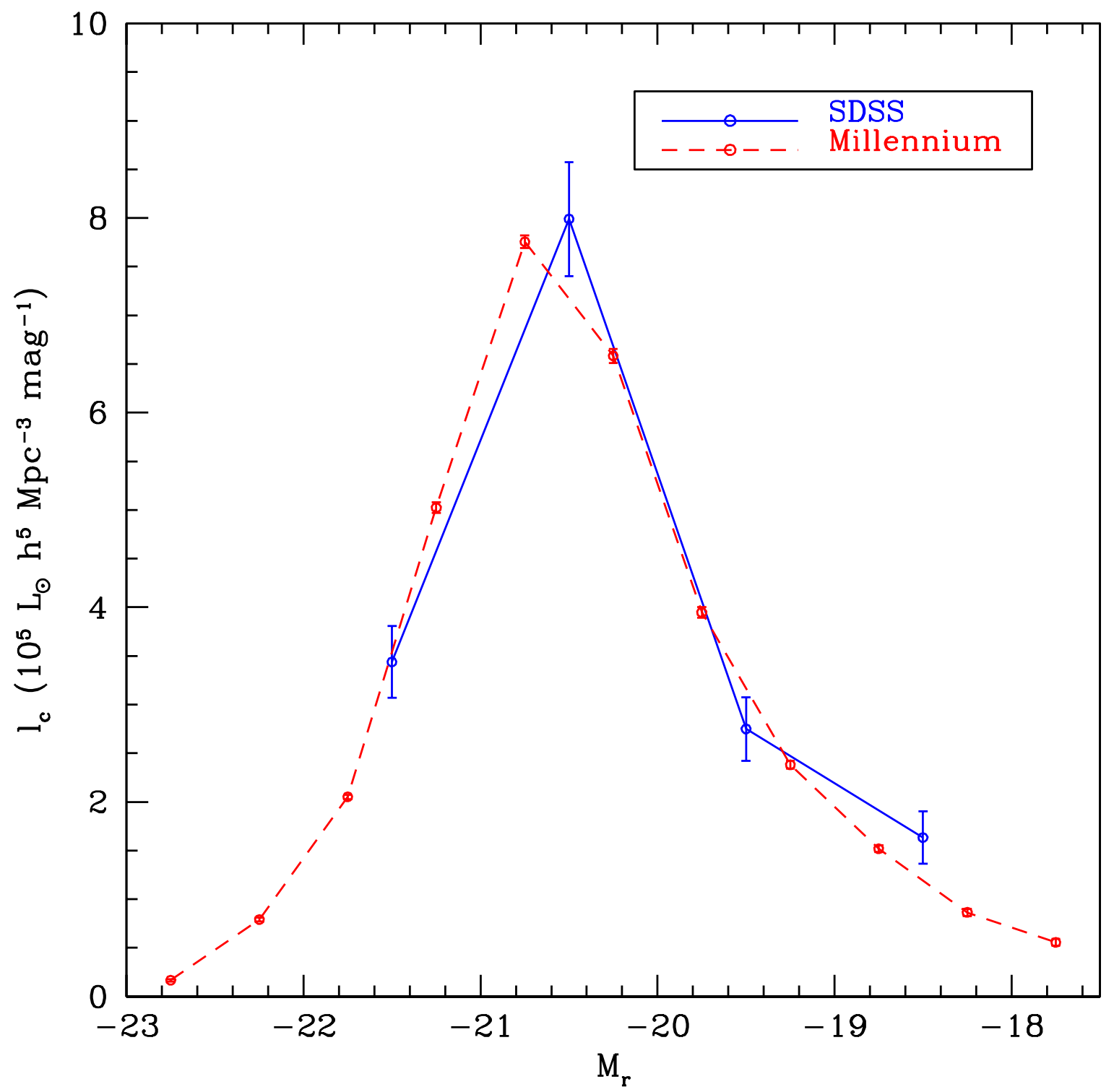

FIG. 9.- The luminosity in close companions per unit co-moving volume $\left(l_{c}\right)$ is plotted versus $M_{r}$ for SDSS and for Millennium. Excellent agreement is seen between the two samples. $l_{c}\left(M_{r}\right)$ exhibits a clear peak at or very close to $M^{*}$. The decline towards brighter absolute magnitudes results primarily from the low number density of such systems, whereas the decline at the faint end results from the decreasing luminosity of companions. In terms of stellar mass (as inferred from galaxy luminosities), this implies that $L^{*}$ is the most relevant regime for major mergers. 